\title{
Article \\ Single-Island Endemism despite Repeated Dispersal in Caribbean Micrathena (Araneae: Araneidae): An Updated Phylogeographic Analysis
}

\author{
Lily Shapiro ${ }^{1, *(D)}$, Greta J. Binford ${ }^{2}$ and Ingi Agnarsson 1,3,* \\ 1 Department of Biology, College of Arts and Sciences, University of Vermont, 109 Carrigan Drive, \\ Burlington, VT 05401, USA \\ 2 Department of Biology, Lewis and Clark College, 615 S. Palatine Hill Road, Portland, OR 97219, USA; \\ binford@lclark.edu \\ 3 Faculty of Life and Environmental Sciences, University of Iceland, Sturlugata 7, 102 Reykjavik, Iceland \\ * Correspondence: lily.shapiro@uvm.edu (L.S.); iagnarsson@gmail.com (I.A.)
}

check for

updates

Citation: Shapiro, L.; Binford, G.J.;

Agnarsson, I. Single-Island

Endemism despite Repeated

Dispersal in Caribbean Micrathena

(Araneae: Araneidae): An Updated

Phylogeographic Analysis. Diversity

2022, 14, 128. https://doi.org/

$10.3390 / \mathrm{d} 14020128$

Academic Editors: Luc Legal and

Matjaž Kuntner

Received: 7 October 2021

Accepted: 5 February 2022

Published: 10 February 2022

Publisher's Note: MDPI stays neutral with regard to jurisdictional claims in published maps and institutional affiliations.

Copyright: (c) 2022 by the authors. Licensee MDPI, Basel, Switzerland. This article is an open access article distributed under the terms and conditions of the Creative Commons Attribution (CC BY) license (https:// creativecommons.org/licenses/by/ $4.0 /)$.

\begin{abstract}
Island biogeographers have long sought to elucidate the mechanisms behind biodiversity genesis. The Caribbean presents a unique stage on which to analyze the diversification process, due to the geologic diversity among the islands and the rich biotic diversity with high levels of island endemism. The colonization of such islands may reflect geologic heterogeneity through vicariant processes and/ or involve long-distance overwater dispersal. Here, we explore the phylogeography of the Caribbean and proximal mainland spiny orbweavers (Micrathena, Araneae), an American spider lineage that is the most diverse in the tropics and is found throughout the Caribbean. We specifically test whether the vicariant colonization via the contested GAARlandia landbridge (putatively emergent 33-35 mya), long-distance dispersal (LDD), or both processes best explain the modern Micrathena distribution. We reconstruct the phylogeny and test biogeographic hypotheses using a 'target gene approach' with three molecular markers (CO1, ITS-2, and 16S rRNA). Phylogenetic analyses support the monophyly of the genus but reject the monophyly of Caribbean Micrathena. Biogeographical analyses support five independent colonizations of the region via multiple overwater dispersal events, primarily from North/Central America, although the genus is South American in origin. There is no evidence for dispersal to the Greater Antilles during the timespan of GAARlandia. Our phylogeny implies greater species richness in the Caribbean than previously known, with two putative species of $M$. forcipata that are each single-island endemics, as well as deep divergences between the Mexican and Floridian M. sagittata. Micrathena is an unusual lineage among arachnids, having colonized the Caribbean multiple times via overwater dispersal after the submergence of GAARlandia. On the other hand, single-island endemism and undiscovered diversity are nearly universal among all but the most dispersal-prone arachnid groups in the Caribbean.
\end{abstract}

Keywords: phylogeny; Caribbean biogeography; GAARlandia; arachnid; araneae; Micrathena; vicariance; long distance dispersal

\section{Introduction}

Understanding the evolutionary machinery of biodiversity genesis in island systems has long been a focus of fundamental biological research [1-4]. Islands serve as discrete, isolated systems in which to study the generation of biodiversity, resulting from complex patterns of (sometimes) repeated colonization, radiation, and extinction. The isolated nature of islands also allows for the evolution of increased magnitudes of endemic forms; archipelagos facilitate these processes, which are replicated continuously across the entire system [5-7]. Such biodiversity is exemplified within Caribbean archipelagoes and can be observed across taxonomic groups, including arthropods, amphibians, fish, mammals, birds, and plants $[7,8]$. The proximity of the Caribbean islands to continental blocks has 
resulted in the production of a unique assemblage of endemic biota, while still being remote enough for the formation of effective oceanic barriers for dispersal [7].

The geologic history of the Caribbean is intrinsically coupled with this biological diversity, and the region itself is composed of islands with varying geologic origins and different regional tectonic influences [9-12]. This complex geology includes old islands such as the Greater Antilles, which have been emergent for at least 40 million years (midEocene) [13] and younger, primarily volcanic islands (e.g., Lesser Antilles) that emerged less than 10 mya (upper Miocene). The distinct geologic history of each island in the Caribbean should be reflected in the modern patterns of organismal diversity, resulting from its colonization via long-distance dispersal and/or vicariant processes, potentially leading to diversification. Newer volcanic islands and isolated limestone/sedimentary oceanic islands, separated from other landmasses by large swaths of ocean, will likely have species assemblages exclusively resulting from long-distance dispersal from the mainland or other island sources. Continental islands, such as the Greater Antilles, are much older island systems with a complex history of islands becoming emergent or submerged, and splintering and rejoining $[12,14,15]$. Unraveling the role of LDD and vicariance for a specific group depends on the geology of an individual island, in conjunction with the biology of that lineage [14-18]. As these islands are deferentially isolated from continents, the dispersal ability of a selected lineage is especially significant in understanding its historical colonization of the Caribbean [19].

The GAARlandia (Greater Antilles Aves Ridge) landbridge is a hypothetical subaerial connection between South America and the Greater Antilles, in which parts of the previously submerged Aves Ridge became exposed as a consequence of dropping sea levels and the Greater Antillean uplift during the Eocene-Oligocene transition (35-33 mya) [20,21]. This ephemeral connection would have permitted direct overland colonization of South American taxa to the Greater Antilles, followed by the subsequent diversification and speciation as organisms filled previously empty niches before the landbridge was resubmerged around 30 mya [20]. The GAARlandia hypothesis, therefore, predicts the simultaneous colonization across diverse taxa to the Greater Antilles within this timespan, a readily testable biological prediction that has recently been evaluated in a variety of Caribbean biogeographic studies across multiple arthropod taxa [14,16,22-36]. While recent chronostratigraphic data suggests the emergence of a landmass between Puerto Rico and the Lesser Antilles in the mid-Eocene, corresponding with crustal shortening and thickening that is consistent with GAARlandia [37], the hypothesis remains contested due to limited [38,39] or conflicting geological and paleo-oceanographic data [40,41]. Ali and Hedges [40], and others cited therein, also emphasize that biogeographic evidence, consistent with the hypothesis, may offer only weak support due to ambiguity in lineage dating. Recent meta-analyses, uniting multiple studies, generally rejected the role of GAARlandia in the biogeography of Caribbean land vertebrates [40], continuing this active debate.

This complex geologic and evolutionary history can be clarified with phylogeographic evidence from densely sampled, regionally-focused clades. Spiders have increasingly been used, in recent years, as biogeographical models not only in the Caribbean but on global and finer scales $[23,42-46]$, as they form a hyperdiverse group with corresponding diversity in dispersal ability and lineage age. While much of the historical research concerning Caribbean biogeography has been vertebrate-based [14,34,47-49], invertebrates, such as arachnids, can provide fine-scale signals of historical dispersal and colonization [16,50]. Recent evidence from these animals have found mixed support for vicariance and LDD, with a large diversity of focal lineages $[16,23,26,29,31,32,36,51,52]$.

Micrathena, the spiny orbweavers (Araneae, Araneidae), are a colorful, highly ornate, and sexually dimorphic group of 119 New World species, distributed from northern Argentina, throughout the Caribbean and Central America, to the New York state, and into southern Ontario [53,54]. Members of the genus reside in forests or woodlands, constructing webs in the understory up to approximately $4 \mathrm{~m}$ off the ground [55]. The large, colorful adult females 
are sedentary and solitary, while the much tinier males wander in search of a mate, preferably a penultimate-instar female (as noted in the case of Micrathena gracilis) [55]. Ballooning behavior has only been formally observed in the juveniles of Micrathena sagittata [56] but the biogeographic patterns $[36,51,53]$ suggest that it may have played a role in overwater dispersal in the Caribbean.

About 67 Micrathena species are South American endemics (most found in Colombia and Brazil), with an additional 25 potentially widespread species that have part of their range in South America [57]. Fourteen species are Central American endemics, and eight are Caribbean endemics. Of the eight Caribbean species, four are known single-island endemics: two from Cuba (M. banksi and M. cubana), one from Jamaica (M. rufopuncata), and one from Hispaniola (M. similis). In addition, Micrathena forcipata from Cuba and Hispaniola, and Micrathena militaris from Puerto Rico and Hispaniola, have recently been suggested to represent clearly divergent lineages, potentially yielding four additional single-island endemics in the Caribbean [51]. Four species are found in North America (M. funebris, M. gracilis, M. mitrata, and M. sagittata), and each of these species is in the Caribbean. A previous phylogeographic analysis of Caribbean Micrathena by McHugh et al. [51] proposed three Caribbean species-groups (the militaris group, the furcula group, and the gracilis group), in agreement with studies by Magalhães et al. [51,53]. Each of these species groups included members of the North, Central, and South American Micrathena, indicating that Caribbean Micrathena are not monophyletic, and that colonization of the Caribbean must have been repetitive [51]. Similar patterns are found in some other members of Araneidae (I. Agnarsson unpublished data).

This paper expands on the work of McHugh et al. [51] with increased taxon sampling of Caribbean Micrathena and additional North and South American mainland species (Colombia and Florida). These additional taxa allow more refined tests of patterns of single-island endemism and more a rigorous evaluation of factors influencing divergence patterns. McHugh et al. [51] rejected the hypothesis that Micrathena colonized the Greater Antilles via the GAARlandia landbridge. Here, we explicitly test the dispersal route using our additional data on previously omitted and undersampled species that help clarify patterns and timelines for the Caribbean colonization in the genus. These tests strengthen our understanding of the continental-island interchange and other biogeographic patterns of Micrathena within the region.

\section{Materials and Methods}

\subsection{Specimen and Taxon Sampling}

Micrathena specimens were collected in the field from 1997-2015 (Table 1, Figure 1). Specimens were stored at $-20{ }^{\circ} \mathrm{C}$ in $95 \%$ ethanol at the University of Vermont. In this work, we added 50 individuals, representing 14 additional Micrathena species, to the previous McHugh et al. [51] Micrathena phylogeography study (M. duodecimspinosa, M. lucasi, M. sp (putative species) M. mitrata, M. beta, M. cornuta, M. embira, M. exlinae, M. miles, $M$. perfida, M. reimoseri, $M$. spinulata, $M$. triangularispinosa, and $M$. yanomami (Table 1$)$ ). We also added previously represented species from new localities: $M$. gracilis from Florida; $M$. horrida from Jamaica; M. militaris from Dominica; M. sagittata from Florida and Mexico; M. schreibersi from Colombia, Trinidad, and Costa Rica; M. sexspinosa from Colombia; and expanded sites of M. forcipata from Cuba, which were sampled on CarBio trips from 2012-2015 (Table 1). We used a specimen of Achaearanea sp. (Theridiidae) as the primary outgroup, along with five araneid members: two Argiope specimens and three Gasteracantha cancriformis individuals. The outgroups included some relatively near relatives of Micrathena [58], along with more distantly related araneid members in Argiope [49], with members of Theridiidae being used to root the tree. 
Table 1. Taxon sampling table with barcodes, locality data, and GenBank accession numbers. " $x$ " denotes GenBank submission in progress.

\begin{tabular}{|c|c|c|c|c|c|c|c|c|}
\hline Genus & Species & Barcode & Country/Region & Latitude & Longitude & $16 S$ & $\mathrm{CO} 1$ & ITS2 \\
\hline Micrathena & annulata & MIC007 & Brazil & $26.08933 S$ & $48.64006 \mathrm{~W}$ & & KJ157272 & \\
\hline Micrathena & aureola & MIC009 & Brazil & 4.904167S & 42.79083W & & KJ157249 & \\
\hline Micrathena & banksi & 784750 & Cuba & $20.05269 \mathrm{~N}$ & $76.50296 \mathrm{~W}$ & KJ156991 & KJ157215 & KJ157104 \\
\hline Micrathena & banksi & 784760 & Cuba & $20.0107 \mathrm{~N}$ & $76.8843 \mathrm{~W}$ & KJ156992 & KJ157216 & \\
\hline Micrathena & banksi & 784976 & Cuba & $20.00939 \mathrm{~N}$ & $76.89402 \mathrm{~W}$ & KJ156993 & KJ157217 & KJ157105 \\
\hline Micrathena & banksi & 785101 & Cuba & $20.00939 \mathrm{~N}$ & $76.89402 \mathrm{~W}$ & KJ156994 & KJ157220 & KJ157106 \\
\hline Micrathena & banksi & 785175 & Cuba & $20.33178 \mathrm{~N}$ & $74.56919 \mathrm{~W}$ & KJ156995 & KJ157219 & KJ157107 \\
\hline Micrathena & banksi & 787933 & Cuba & $20.01742 \mathrm{~N}$ & $76.89781 \mathrm{~W}$ & KJ156996 & KJ157218 & KJ157108 \\
\hline Micrathena & beta & MIC238 & Peru & $4.5674444 S$ & $73.45925 \mathrm{~W}$ & & KX687306 & \\
\hline Micrathena & bimucronata & MIC123 & Costa Rica & $10.233518 \mathrm{~N}$ & 84.075411W & & KJ157236 & \\
\hline Micrathena & brevipes & MIC121 & Costa Rica & $9.552960 \mathrm{~N}$ & 83.112910W & & KJ157223 & \\
\hline Micrathena & cornuta & MIC199 & Peru & $12.8088056 \mathrm{~S}$ & 69.30175W & & KX687309 & \\
\hline Micrathena & cubana & 784355 & Cuba & $20.01309 \mathrm{~N}$ & $76.83400 \mathrm{~W}$ & KJ156997 & KJ157224 & KJ157109 \\
\hline Micrathena & cubana & 784820 & Cuba & $20.00874 \mathrm{~N}$ & 76.88777W & KJ156998 & KJ157225 & KJ157110 \\
\hline Micrathena & cubana & 785048 & Cuba & $22.65707 \mathrm{~N}$ & 83.70161W & KJ156999 & KJ157226 & KJ157111 \\
\hline Micrathena & cubana & 787840 & Cuba & $20.33178 \mathrm{~N}$ & $74.56919 \mathrm{~W}$ & KJ157000 & KJ157227 & \\
\hline Micrathena & digitata & MIC017 & Brazil & $11.39983 S$ & $40.52206 \mathrm{~W}$ & & KJ157238 & \\
\hline Micrathena & duodecimspinosa & 00004833A & Costa Rica & $\begin{array}{c}\text { San Antonio de } \\
\text { Escazú }\end{array}$ & & & $\mathrm{x}$ & $\mathrm{x}$ \\
\hline Micrathena & embira & MIC182 & Brazil & $9.642419 S$ & $41.446727 \mathrm{~W}$ & & KX687311 & \\
\hline Micrathena & exlinae & MIC147 & Brazil & $0.99185 S$ & $62.15915 \mathrm{~W}$ & & KX687313 & \\
\hline Micrathena & forcipata & 00002846A & Cuba & $\begin{array}{c}\text { Juan Gonzalez, } \\
\text { Guamá }\end{array}$ & & & $\mathrm{x}$ & $x$ \\
\hline Micrathena & forcipata & 00002848A & Cuba & $20.01309 \mathrm{~N}$ & $76.83400 \mathrm{~W}$ & & $x$ & $x$ \\
\hline Micrathena & forcipata & 00002845A & Cuba & $20.01309 \mathrm{~N}$ & $76.83400 \mathrm{~W}$ & & $x$ & $x$ \\
\hline Micrathena & forcipata & 784425 & Cuba & $20.00939 \mathrm{~N}$ & $76.89402 \mathrm{~W}$ & KJ157002 & KJ157256 & KJ157113 \\
\hline Micrathena & forcipata & 787842 & Cuba & $20.33178 \mathrm{~N}$ & $74.56919 \mathrm{~W}$ & KJ157003 & KJ157257 & \\
\hline Micrathena & forcipata & 782311 & Hispaniola & $18.355536 \mathrm{~N}$ & $68.61825 \mathrm{~W}$ & KJ157004 & KJ157258 & \\
\hline Micrathena & forcipata & 782434 & Hispaniola & $19.34405 \mathrm{~N}$ & $69.46635 \mathrm{~W}$ & KJ157005 & KJ157260 & KJ157114 \\
\hline Micrathena & forcipata & 784362 & Hispaniola & $18.32902 \mathrm{~N}$ & 68.80995W & KJ157006 & KJ157264 & KJ157115 \\
\hline Micrathena & forcipata & 784366 & Hispaniola & $18.32902 \mathrm{~N}$ & $68.80995 \mathrm{~W}$ & & KJ157271 & KJ157116 \\
\hline Micrathena & forcipata & 784447 & Hispaniola & $18.2205360 \mathrm{~N}$ & $68.480607 \mathrm{~W}$ & KJ157007 & KJ157261 & KJ157117 \\
\hline Micrathena & forcipata & 785054 & Hispaniola & $19.746175 \mathrm{~N}$ & $71.257726 \mathrm{~W}$ & KJ157008 & KJ157263 & KJ157118 \\
\hline Micrathena & forcipata & 785282 & Hispaniola & $18.355536 \mathrm{~N}$ & $68.6185 \mathrm{~W}$ & KJ157009 & KJ157259 & KJ157119 \\
\hline Micrathena & forcipata & 785682 & Hispaniola & $18.2205360 \mathrm{~N}$ & $68.480607 \mathrm{~W}$ & KJ157010 & KJ157 & \\
\hline Micrathena & forcipata & 787132 & Hispaniola & $18.310010 \mathrm{~N}$ & $71.6000 \mathrm{~W}$ & & KJ157265 & \\
\hline Micrathena & forcipata & 787135 & Hispaniola & $18.310010 \mathrm{~N}$ & $71.6000 \mathrm{~W}$ & KJ157011 & KJ157266 & \\
\hline Micrathena & forcipata & 787150 & Hispaniola & $18.310010 \mathrm{~N}$ & $71.6000 \mathrm{~W}$ & KJ157012 & KJ157267 & KJ157121 \\
\hline Micrathena & forcipata & 787153 & Hispaniola & $18.310010 \mathrm{~N}$ & $71.6000 \mathrm{~W}$ & KJ157013 & KJ157269 & KJ157122 \\
\hline Micrathena & forcipata & 787210 & Hispaniola & $18.310010 \mathrm{~N}$ & $71.6000 \mathrm{~W}$ & KJ157014 & KJ157268 & KJ157123 \\
\hline Micrathena & forcipata & 787243 & Hispaniola & $18.310010 \mathrm{~N}$ & $71.6000 \mathrm{~W}$ & KJ157015 & KJ157270 & KJ157124 \\
\hline
\end{tabular}


Table 1. Cont.

\begin{tabular}{|c|c|c|c|c|c|c|c|c|}
\hline Genus & Species & Barcode & Country/Region & Latitude & Longitude & $16 S$ & CO1 & ITS2 \\
\hline Micrathena & furcata & MIC037 & Brazil & $27.66667 \mathrm{~S}$ & $49.01667 \mathrm{~W}$ & & KJ157242 & \\
\hline Micrathena & gracilis & 10000619A & FL, USA & $29.4776 \mathrm{~N}$ & $82.5627 \mathrm{~W}$ & & $x$ & $x$ \\
\hline Micrathena & gracilis & $10000629 \mathrm{~A}$ & FL, USA & $29.62986 \mathrm{~N}$ & $82.29880 \mathrm{~W}$ & & $x$ & \\
\hline Micrathena & gracilis & 10000627A & FL, USA & $29.62986 \mathrm{~N}$ & $82.29880 \mathrm{~W}$ & & $x$ & \\
\hline Micrathena & gracilis & $10000638 \mathrm{~A}$ & FL, USA & $29.63680 \mathrm{~N}$ & $82.23961 \mathrm{~W}$ & & $x$ & $x$ \\
\hline Micrathena & gracilis & $10000644 \mathrm{~A}$ & FL, USA & $29.46368 \mathrm{~N}$ & $82.52898 \mathrm{~W}$ & & $x$ & \\
\hline Micrathena & gracilis & $10000642 \mathrm{~A}$ & FL, USA & $29.62688 \mathrm{~N}$ & $82.29878 \mathrm{~W}$ & & $x$ & \\
\hline Micrathena & gracilis & $10000643 \mathrm{~A}$ & FL, USA & $29.62688 \mathrm{~N}$ & $82.29878 \mathrm{~W}$ & & $x$ & \\
\hline Micrathena & gracilis & 00000804A & NC, USA & $35.44842 \mathrm{~N}$ & $81.58694 \mathrm{~W}$ & & KJ157250 & KJ157188 \\
\hline Micrathena & gracilis & 00000954A & SC, USA & $33.03913 \mathrm{~N}$ & $79.56459 \mathrm{~W}$ & KJ157084 & KJ157252 & KJ157192 \\
\hline Micrathena & gracilis & 00000935A & SC, USA & $33.03913 \mathrm{~N}$ & $79.56459 \mathrm{~W}$ & KJ157083 & KJ157254 & KJ157191 \\
\hline Micrathena & gracilis & 00000889A & SC, USA & $33.03913 \mathrm{~N}$ & $79.56459 \mathrm{~W}$ & KJ157082 & KJ157251 & KJ157190 \\
\hline Micrathena & gracilis & 00000984A & SC, USA & $33.03913 \mathrm{~N}$ & $79.56459 \mathrm{~W}$ & KJ157086 & KJ157253 & KJ157194 \\
\hline Micrathena & gracilis & 00000988A & SC, USA & $33.03913 \mathrm{~N}$ & 79.56459W & KJ157087 & KJ157255 & KJ157195 \\
\hline Micrathena & gracilis & 00002487A & NY, USA & $42.01807 \mathrm{~N}$ & 73.91707W & KJ157088 & & KJ157196 \\
\hline Micrathena & gracilis & 00002501A & NY, USA & $42.01807 \mathrm{~N}$ & 73.91707W & KJ157089 & & KJ157197 \\
\hline Micrathena & gracilis & 00000976A & SC, USA & $33.03913 \mathrm{~N}$ & $79.56459 \mathrm{~W}$ & KJ157085 & & KJ157193 \\
\hline Micrathena & horrida & MIC042 & Brazil & $16.59553 S$ & $41.57925 \mathrm{~W}$ & & KJ157248 & \\
\hline Micrathena & horrida & MIC122 & Costa Rica & $10.233518 \mathrm{~N}$ & 84.075411W & & KJ157245 & \\
\hline Micrathena & horrida & 00003552A & Jamaica & $18.1635 \mathrm{~N}$ & 77.39410W & & $x$ & $x$ \\
\hline Micrathena & horrida & 784351 & Cuba & $20.00939 \mathrm{~N}$ & $76.89402 \mathrm{~W}$ & KJ157016 & KJ157243 & KJ157125 \\
\hline Micrathena & horrida & 784751 & Cuba & $20.00939 \mathrm{~N}$ & $76.89402 \mathrm{~W}$ & KJ157017 & KJ157246 & KJ157126 \\
\hline Micrathena & horrida & 787913 & Cuba & $20.00939 \mathrm{~N}$ & $76.89402 W$ & KJ157018 & KJ157247 & KJ157127 \\
\hline Micrathena & horrida & 787919 & Cuba & $20.00939 \mathrm{~N}$ & $76.89402 W$ & KJ157019 & KJ157244 & KJ157128 \\
\hline Micrathena & lucasi & 00004785A & Costa Rica & $\begin{array}{c}\text { San Antonio de } \\
\text { Escazú }\end{array}$ & & & & \\
\hline Micrathena & macfarlanei & MIC054 & Brazil & $19.65000 \mathrm{~S}$ & $42.56667 \mathrm{~W}$ & & KJ157241 & \\
\hline Micrathena & miles & MIC142 & Peru & $3.82975 \mathrm{~S}$ & 73.375333W & & KX687317 & \\
\hline Micrathena & militaris & $10000526 \mathrm{~A}$ & Dominica & $15.32710 \mathrm{~N}$ & $61.3381 \mathrm{~W}$ & & $x$ & $x$ \\
\hline Micrathena & militaris & $10000528 \mathrm{~A}$ & Dominica & $15.32710 \mathrm{~N}$ & $61.3381 \mathrm{~W}$ & & $x$ & $x$ \\
\hline Micrathena & militaris & 782365 & Hispaniola & $18.355536 \mathrm{~N}$ & 068.61825W & KJ157020 & & KJ157129 \\
\hline Micrathena & militaris & 784338 & Hispaniola & $18.32902 \mathrm{~N}$ & 068.80995W & KJ157021 & KJ157273 & \\
\hline Micrathena & militaris & 784363 & Hispaniola & $18.32902 \mathrm{~N}$ & 068.80995W & KJ157022 & KJ157293 & KJ157130 \\
\hline Micrathena & militaris & 784403 & Hispaniola & $18.32902 \mathrm{~N}$ & 068.80995W & KJ157023 & KJ157298 & KJ157131 \\
\hline Micrathena & militaris & 784430 & Hispaniola & $18.32902 \mathrm{~N}$ & 068.80995W & KJ157024 & & KJ157132 \\
\hline Micrathena & militaris & 784448 & Hispaniola & $18.32902 \mathrm{~N}$ & 068.80995W & KJ157025 & KJ157294 & KJ157133 \\
\hline Micrathena & militaris & 784458 & Hispaniola & $18.32902 \mathrm{~N}$ & 068.80995W & KJ157026 & & KJ157134 \\
\hline Micrathena & militaris & 784503 & Hispaniola & $18.3150011 \mathrm{~N}$ & 71.580556W & KJ157027 & KJ157300 & KJ157135 \\
\hline Micrathena & militaris & 784531 & Hispaniola & $18.355536 \mathrm{~N}$ & 068.61825W & KJ157028 & & KJ157136 \\
\hline Micrathena & militaris & 784566 & Hispaniola & $18.32902 \mathrm{~N}$ & 068.80995W & KJ157029 & KJ157296 & KJ157137 \\
\hline Micrathena & militaris & 784671 & Hispaniola & $19.06707 \mathrm{~N}$ & $069.46355 \mathrm{~W}$ & KJ157030 & & KJ157138 \\
\hline Micrathena & militaris & 784721 & Hispaniola & $18.32902 \mathrm{~N}$ & 068.80995W & KJ157031 & KJ157310 & KJ157139 \\
\hline
\end{tabular}


Table 1. Cont.

\begin{tabular}{|c|c|c|c|c|c|c|c|c|}
\hline Genus & Species & Barcode & Country/Region & Latitude & Longitude & $16 S$ & CO1 & ITS2 \\
\hline Micrathena & militaris & 784759 & Hispaniola & $18.355536 \mathrm{~N}$ & $068.61825 \mathrm{~W}$ & KJ157032 & KJ157277 & KJ157140 \\
\hline Micrathena & militaris & 784762 & Hispaniola & $18.2205360 \mathrm{~N}$ & 68.4806070W & KJ157033 & & KJ157141 \\
\hline Micrathena & militaris & 784772 & Hispaniola & $18.32902 \mathrm{~N}$ & 068.80995W & KJ157034 & KJ157287 & KJ157142 \\
\hline Micrathena & militaris & 784806 & Hispaniola & & & KJ157035 & & KJ157143 \\
\hline Micrathena & militaris & 784926 & Hispaniola & & & KJ157036 & & KJ157144 \\
\hline Micrathena & militaris & 785066 & Hispaniola & $19.06707 \mathrm{~N}$ & $069.46355 \mathrm{~W}$ & KJ157037 & & KJ157145 \\
\hline Micrathena & militaris & 785080 & Hispaniola & $18.32902 \mathrm{~N}$ & 068.80995W & KJ157038 & KJ157274 & KJ157146 \\
\hline Micrathena & militaris & 785099 & Hispaniola & $18.32902 \mathrm{~N}$ & 068.80995W & & KJ157313 & \\
\hline Micrathena & militaris & 785128 & Hispaniola & $18.355536 \mathrm{~N}$ & 068.61825W & KJ157039 & & KJ157147 \\
\hline Micrathena & militaris & 785144 & Hispaniola & $19.746175 \mathrm{~N}$ & 71.257726W & KJ157040 & & KJ157148 \\
\hline Micrathena & militaris & 785169 & Hispaniola & $18.355536 \mathrm{~N}$ & 068.61825W & KJ157041 & KJ157290 & KJ157149 \\
\hline Micrathena & militaris & 785173 & Hispaniola & $19.06707 \mathrm{~N}$ & 069.46355W & KJ157042 & KJ157314 & KJ157150 \\
\hline Micrathena & militaris & 785174 & Hispaniola & $19.06707 \mathrm{~N}$ & $069.46355 \mathrm{~W}$ & KJ157043 & KJ157292 & KJ157151 \\
\hline Micrathena & militaris & 785194 & Hispaniola & $18.355536 \mathrm{~N}$ & 068.61825W & KJ157044 & & \\
\hline Micrathena & militaris & 785208 & Hispaniola & $18.2205360 \mathrm{~N}$ & $68.4806070 \mathrm{~W}$ & KJ157045 & KJ157297 & KJ157152 \\
\hline Micrathena & militaris & 785219 & Hispaniola & $18.355536 \mathrm{~N}$ & 068.61825W & KJ157046 & KJ157286 & KJ157153 \\
\hline Micrathena & militaris & 785263 & Hispaniola & $18.355536 \mathrm{~N}$ & 068.61825W & KJ157047 & & KJ157154 \\
\hline Micrathena & militaris & 785273 & Hispaniola & $19.432213 \mathrm{~N}$ & 070.371412W & KJ157048 & KJ157275 & KJ157155 \\
\hline Micrathena & militaris & 785280 & Hispaniola & $18.32902 \mathrm{~N}$ & 068.80995W & KJ157049 & KJ157315 & KJ157156 \\
\hline Micrathena & militaris & 785312 & Hispaniola & $19.34405 \mathrm{~N}$ & 069.46635W & KJ157050 & KJ157280 & KJ157157 \\
\hline Micrathena & militaris & 785401 & Hispaniola & $19.06707 \mathrm{~N}$ & $069.46355 \mathrm{~W}$ & KJ157051 & KJ157276 & KJ157158 \\
\hline Micrathena & militaris & 785402 & Hispaniola & $19.34405 \mathrm{~N}$ & $069.46635 \mathrm{~W}$ & KJ157052 & KJ157285 & KJ157159 \\
\hline Micrathena & militaris & 785423 & Hispaniola & $18.355536 \mathrm{~N}$ & 068.61825W & KJ157053 & & KJ157160 \\
\hline Micrathena & militaris & 785461 & Hispaniola & $19.06707 \mathrm{~N}$ & $069.46355 \mathrm{~W}$ & KJ157054 & KJ157281 & \\
\hline Micrathena & militaris & 785502 & Hispaniola & $19.06707 \mathrm{~N}$ & $069.46355 \mathrm{~W}$ & KJ157055 & KJ157301 & KJ157161 \\
\hline Micrathena & militaris & 785512 & Hispaniola & $19.06707 \mathrm{~N}$ & $069.46355 \mathrm{~W}$ & KJ157056 & KJ157316 & KJ157162 \\
\hline Micrathena & militaris & 785524 & Hispaniola & $18.355536 \mathrm{~N}$ & 068.61825W & KJ157057 & KJ157311 & KJ157163 \\
\hline Micrathena & militaris & 785527 & Hispaniola & $19.34405 \mathrm{~N}$ & $069.46635 \mathrm{~W}$ & KJ157058 & KJ157279 & KJ157164 \\
\hline Micrathena & militaris & 785563 & Hispaniola & $19.06707 \mathrm{~N}$ & $069.46355 \mathrm{~W}$ & KJ157059 & KJ157295 & KJ157165 \\
\hline Micrathena & militaris & 785604 & Hispaniola & $19.06707 \mathrm{~N}$ & 069.46355W & KJ157060 & KJ157288 & KJ157166 \\
\hline Micrathena & militaris & 785706 & Hispaniola & $19.06707 \mathrm{~N}$ & 069.46355W & KJ157061 & KJ157278 & KJ157167 \\
\hline Micrathena & militaris & 785709 & Hispaniola & $19.06707 \mathrm{~N}$ & $069.46355 \mathrm{~W}$ & & KJ157312 & KJ157168 \\
\hline Micrathena & militaris & 785722 & Hispaniola & $19.06707 \mathrm{~N}$ & $069.46355 \mathrm{~W}$ & KJ157062 & KJ157283 & KJ157169 \\
\hline Micrathena & militaris & 785729 & Hispaniola & $19.34405 \mathrm{~N}$ & $069.46635 \mathrm{~W}$ & KJ157063 & KJ157284 & KJ157170 \\
\hline Micrathena & militaris & 785743 & Hispaniola & $19.06707 \mathrm{~N}$ & 069.46355W & KJ157064 & KJ157282 & KJ157171 \\
\hline Micrathena & militaris & 785769 & Hispaniola & $19.06707 \mathrm{~N}$ & 069.46355W & KJ157065 & & KJ157172 \\
\hline Micrathena & militaris & 787068 & Hispaniola & $18.980122 \mathrm{~N}$ & $70.798425 \mathrm{~W}$ & KJ157066 & KJ157299 & KJ157173 \\
\hline Micrathena & militaris & 787106 & Hispaniola & $18.980122 \mathrm{~N}$ & $70.798425 \mathrm{~W}$ & KJ157067 & KJ157289 & KJ157174 \\
\hline Micrathena & militaris & 787148 & Hispaniola & $18.3150011 \mathrm{~N}$ & 71.580556W & KJ157068 & KJ157291 & KJ157175 \\
\hline Micrathena & militaris & 787152 & Hispaniola & $18.3150011 \mathrm{~N}$ & 71.580556W & KJ157069 & & KJ157176 \\
\hline Micrathena & militaris & 787166 & Hispaniola & $18.3150011 \mathrm{~N}$ & 71.580556W & KJ157070 & & KJ157177 \\
\hline Micrathena & militaris & 787190 & Hispaniola & $18.3150011 \mathrm{~N}$ & 71.580556W & KJ157071 & & KJ157178 \\
\hline
\end{tabular}


Table 1. Cont.

\begin{tabular}{|c|c|c|c|c|c|c|c|c|}
\hline Genus & Species & Barcode & Country/Region & Latitude & Longitude & $16 S$ & CO1 & ITS2 \\
\hline Micrathena & militaris & 787208 & Hispaniola & $18.3150011 \mathrm{~N}$ & $71.580556 \mathrm{~W}$ & KJ157072 & & KJ157179 \\
\hline Micrathena & militaris & 787212 & Hispaniola & $18.3150011 \mathrm{~N}$ & $71.580556 \mathrm{~W}$ & KJ157073 & & KJ157180 \\
\hline Micrathena & militaris & 787214 & Hispaniola & $18.3150011 \mathrm{~N}$ & $71.580556 \mathrm{~W}$ & KJ157001 & & KJ157112 \\
\hline Micrathena & militaris & 392672 & Puerto Rico & $17.971472 \mathrm{~N}$ & 66.867958W & KJ157074 & KJ157302 & KJ157181 \\
\hline Micrathena & militaris & 392677 & Puerto Rico & $17.971472 \mathrm{~N}$ & 66.867958W & KJ157075 & KJ157303 & KJ157182 \\
\hline Micrathena & militaris & 782048 & Puerto Rico & $18.414373 \mathrm{~N}$ & $66.728722 \mathrm{~W}$ & KJ157076 & KJ157307 & KJ157183 \\
\hline Micrathena & militaris & 782126 & Puerto Rico & $18.173264 \mathrm{~N}$ & $66.590149 \mathrm{~W}$ & KJ157077 & KJ157308 & KJ157184 \\
\hline Micrathena & militaris & 782153 & Puerto Rico & $18.414373 \mathrm{~N}$ & $66.728722 \mathrm{~W}$ & KJ157078 & KJ157306 & KJ157185 \\
\hline Micrathena & militaris & 782174 & Puerto Rico & $18.414373 \mathrm{~N}$ & $66.728722 \mathrm{~W}$ & KJ157079 & KJ157304 & KJ157186 \\
\hline Micrathena & militaris & 782201 & Puerto Rico & $18.032518 \mathrm{~N}$ & $67.094653 \mathrm{~W}$ & KJ157080 & KJ157305 & KJ157187 \\
\hline Micrathena & militaris & 783400 & Puerto Rico & $18.45226 \mathrm{~N}$ & 66.59711W & & KJ157309 & \\
\hline Micrathena & mitrata & $10000679 \mathrm{~A}$ & Mexico & $19.79357 \mathrm{~N}$ & $104.0554 \mathrm{~W}$ & & $x$ & $x$ \\
\hline Micrathena & mitrata & 00002849A & Mexico & $19.79357 \mathrm{~N}$ & $104.0554 \mathrm{~W}$ & & $\mathrm{x}$ & $x$ \\
\hline Micrathena & nigrichelis & MIC056 & Brazil & $20.43481 S$ & $43.50906 \mathrm{~W}$ & & KJ157239 & \\
\hline Micrathena & perfida & MIC026 & Brazil & $24.387111 S$ & 47.017583W & & KX687318 & \\
\hline Micrathena & plana & MIC062 & Brazil & $16.53294 S$ & $41.51042 \mathrm{~W}$ & & KJ157240 & \\
\hline Micrathena & reimoseri & MIC072 & Brazil & $11.399833 S$ & 40.522056W & & KX687321 & \\
\hline Micrathena & saccata & MIC076 & Brazil & $1.424828 \mathrm{~S}$ & $48.43802 \mathrm{~W}$ & & KJ157237 & \\
\hline Micrathena & sagittata & $10000618 \mathrm{~A}$ & FL, USA & $29.4776 \mathrm{~N}$ & $082.5627 \mathrm{~W}$ & & $\mathrm{x}$ & \\
\hline Micrathena & sagittata & $10000621 \mathrm{~A}$ & FL, USA & $29.63703 \mathrm{~N}$ & 082.23976W & & $x$ & \\
\hline Micrathena & sagittata & $10000631 \mathrm{~A}$ & FL, USA & $29.62986 \mathrm{~N}$ & 082.29880W & & $x$ & $x$ \\
\hline Micrathena & sagittata & $10000633 \mathrm{~A}$ & FL, USA & $29.62986 \mathrm{~N}$ & 082.29880W & & $x$ & \\
\hline Micrathena & sagittata & $10000636 \mathrm{~A}$ & FL, USA & $29.63680 \mathrm{~N}$ & 082.23961W & & $\mathrm{x}$ & $x$ \\
\hline Micrathena & sagittata & $10000634 \mathrm{~A}$ & FL, USA & $29.46397 \mathrm{~N}$ & $082.55285 \mathrm{~W}$ & & $x$ & $x$ \\
\hline Micrathena & sagittata & 10000639A & FL, USA & $29.63680 \mathrm{~N}$ & $082.23961 \mathrm{~W}$ & & $x$ & \\
\hline Micrathena & sagittata & $10000640 \mathrm{~A}$ & FL, USA & $29.62688 \mathrm{~N}$ & 082.29878W & & $x$ & \\
\hline Micrathena & sagittata & 00002847A & Mexico & $18.18963 \mathrm{~N}$ & $89.46333 \mathrm{~W}$ & & $x$ & \\
\hline Micrathena & sagittata & 00000833A & SC, USA & $33.03913 \mathrm{~N}$ & $79.56459 \mathrm{~W}$ & KJ157081 & KJ157221 & KJ157189 \\
\hline Micrathena & schreibersi & 00002357A & Colombia & Bucaramanga & & & $x$ & \\
\hline Micrathena & schreibersi & $10000650 \mathrm{~A}$ & Colombia & $8.39104 \mathrm{~N}$ & 77.21548W & & $x$ & \\
\hline Micrathena & schreibersi & $10000652 \mathrm{~A}$ & Colombia & $8.39104 \mathrm{~N}$ & 77.21548W & & $x$ & \\
\hline Micrathena & schreibersi & $10000653 \mathrm{~A}$ & Colombia & $8.39104 \mathrm{~N}$ & 77.21548W & & $x$ & $x$ \\
\hline Micrathena & schreibersi & $10000664 \mathrm{~A}$ & Colombia & $8.424 \mathrm{~N}$ & 77.29216W & & $x$ & \\
\hline Micrathena & schreibersi & $10000673 \mathrm{~A}$ & Colombia & $8.39104 \mathrm{~N}$ & 77.21548W & & $x$ & \\
\hline Micrathena & schreibersi & $10000658 \mathrm{~A}$ & Colombia & $8.39104 \mathrm{~N}$ & 77.21548W & & $\mathrm{x}$ & \\
\hline Micrathena & schreibersi & $10000651 \mathrm{~A}$ & Colombia & $8.39104 \mathrm{~N}$ & 77.21548W & & $x$ & $x$ \\
\hline Micrathena & schreibersi & $10000663 \mathrm{~A}$ & Colombia & $8.424 \mathrm{~N}$ & 77.29216W & & $x$ & \\
\hline Micrathena & schreibersi & $10000665 \mathrm{~A}$ & Colombia & $8.424 \mathrm{~N}$ & 77.29216W & & $x$ & $x$ \\
\hline Micrathena & schreibersi & 00004787A & Colombia & $10.21192 \mathrm{~N}$ & $75.25403 \mathrm{~W}$ & & $x$ & $x$ \\
\hline Micrathena & schreibersi & 00004818A & Trinidad & & & & $\mathrm{x}$ & $x$ \\
\hline Micrathena & schreibersi & 00002900A & Costa Rica & $10.430686 \mathrm{~N}$ & $84.007089 \mathrm{~W}$ & & $x$ & $x$ \\
\hline Micrathena & schreibersi & 00000936A & Colombia & $7.062695 \mathrm{~N}$ & 73.073058W & KJ157090 & KJ157318 & KJ157198 \\
\hline Micrathena & schreibersi & 00002357A & Colombia & $7.062695 \mathrm{~N}$ & 73.073058W & KJ157092 & KJ157319 & KJ157199 \\
\hline
\end{tabular}


Table 1. Cont.

\begin{tabular}{|c|c|c|c|c|c|c|c|c|}
\hline Genus & Species & Barcode & Country/Region & Latitude & Longitude & $16 S$ & $\mathrm{CO} 1$ & ITS2 \\
\hline Micrathena & sexspinosa & $10000690 \mathrm{~A}$ & Colombia & $8.35249 \mathrm{~N}$ & 77.22118W & & $x$ & \\
\hline Micrathena & sexspinosa & 10000659A & Colombia & $8.35249 \mathrm{~N}$ & 77.22118W & & $\mathrm{x}$ & \\
\hline Micrathena & sexspinosa & $10000674 \mathrm{~A}$ & Colombia & $8.35249 \mathrm{~N}$ & $77.22118 \mathrm{~W}$ & & $x$ & $x$ \\
\hline Micrathena & sexspinosa & 10000677A & Colombia & $11.120083 \mathrm{~N}$ & $74.082805 \mathrm{~W}$ & & $x$ & \\
\hline Micrathena & sexspinosa & $10000683 \mathrm{~A}$ & Colombia & $11.120083 \mathrm{~N}$ & $74.082805 \mathrm{~W}$ & & $x$ & \\
\hline Micrathena & sexspinosa & 10000669A & Colombia & $8.39104 \mathrm{~N}$ & $77.21548 \mathrm{~W}$ & & $\mathrm{x}$ & $x$ \\
\hline Micrathena & sexspinosa & $10000670 \mathrm{~A}$ & Colombia & $8.39104 \mathrm{~N}$ & 77.21548W & & $x$ & $x$ \\
\hline Micrathena & sexspinosa & $10000681 \mathrm{~A}$ & Colombia & $8.35249 \mathrm{~N}$ & $77.22118 \mathrm{~W}$ & & $x$ & \\
\hline Micrathena & sexspinosa & $10000678 \mathrm{~A}$ & Colombia & $8.35249 \mathrm{~N}$ & $77.22118 \mathrm{~W}$ & & $\mathrm{x}$ & \\
\hline Micrathena & sexspinosa & 00000987A & Colombia & $7.062695 \mathrm{~N}$ & 73.073058W & KJ157091 & KJ157222 & \\
\hline Micrathena & similis & 785024 & Hispaniola & $19.34405 \mathrm{~N}$ & $69.46635 \mathrm{~W}$ & KJ157093 & KJ157228 & KJ157200 \\
\hline Micrathena & similis & 785496 & Hispaniola & $19.34405 \mathrm{~N}$ & $69.46635 \mathrm{~W}$ & KJ157094 & KJ157232 & KJ157201 \\
\hline Micrathena & similis & 787265 & Hispaniola & $19.05116 \mathrm{~N}$ & $70.88866 \mathrm{~W}$ & KJ157095 & KJ157233 & KJ157202 \\
\hline Micrathena & similis & 787297 & Hispaniola & $19.05116 \mathrm{~N}$ & $70.88866 \mathrm{~W}$ & KJ157096 & & KJ157203 \\
\hline Micrathena & similis & 787308 & Hispaniola & $19.03627 \mathrm{~N}$ & 70.54337W & KJ157097 & KJ157229 & KJ157204 \\
\hline Micrathena & similis & 787309 & Hispaniola & $19.05116 \mathrm{~N}$ & $70.88866 \mathrm{~W}$ & KJ157098 & & KJ157205 \\
\hline Micrathena & similis & 787311 & Hispaniola & $19.05116 \mathrm{~N}$ & 70.88866W & & KJ157235 & KJ157206 \\
\hline Micrathena & similis & 787318 & Hispaniola & $19.03627 \mathrm{~N}$ & 70.54337W & KJ157099 & KJ157234 & KJ157207 \\
\hline Micrathena & similis & 787320 & Hispaniola & $19.05116 \mathrm{~N}$ & $70.88866 \mathrm{~W}$ & KJ157100 & KJ157230 & KJ157208 \\
\hline Micrathena & similis & 787322 & Hispaniola & $19.05116 \mathrm{~N}$ & $70.88866 \mathrm{~W}$ & KJ157101 & KJ157231 & KJ157209 \\
\hline Micrathena & sp. & $10000656 \mathrm{~A}$ & Colombia & $11.120083 \mathrm{~N}$ & $74.082805 \mathrm{~W}$ & & $x$ & \\
\hline Micrathena & sp. & $10000671 \mathrm{~A}$ & Colombia & $11.120083 \mathrm{~N}$ & $74.082805 \mathrm{~W}$ & & $x$ & $x$ \\
\hline Micrathena & sp. & 00006693A & Colombia & $11.120083 \mathrm{~N}$ & $74.082805 \mathrm{~W}$ & & $x$ & $x$ \\
\hline Micrathena & spinulata & MIC205 & Mexico & $19.1381667 \mathrm{~N}$ & $97.2045 \mathrm{~W}$ & & KX687324 & \\
\hline Micrathena & triangularispinosa & MIC156 & Brazil & $0.97799 S$ & $62.10292 \mathrm{~W}$ & & KX687327 & \\
\hline Micrathena & yanomami & MIC193 & Peru & 13.055639S & $71.546194 W$ & & KX687332 & \\
\hline \multicolumn{9}{|l|}{ Outgroups } \\
\hline Achaearanea & sp. & 784841 & Cuba & $21.59166 \mathrm{~N}$ & $77.78822 \mathrm{~W}$ & & KJ157211 & \\
\hline Argiope & lobata & Arg0160 & Spain & $\begin{array}{c}\text { Missing GPS } \\
\text { data }\end{array}$ & & KJ156988 & & KJ157103 \\
\hline Gasteracantha & cancriformis & 787198 & Hispaniola & $18.3150011 \mathrm{~N}$ & $71.580556 \mathrm{~W}$ & KJ156989 & KJ157212 & \\
\hline Gasteracantha & cancriformis & 784515 & Hispaniola & $18.2205260 \mathrm{~N}$ & $68.480607 \mathrm{~W}$ & & KJ157213 & \\
\hline Gasteracantha & cancriformis & 782149 & Puerto Rico & 18. $172979 \mathrm{~N}$ & 66.491798W & KJ156990 & KJ157214 & \\
\hline
\end{tabular}




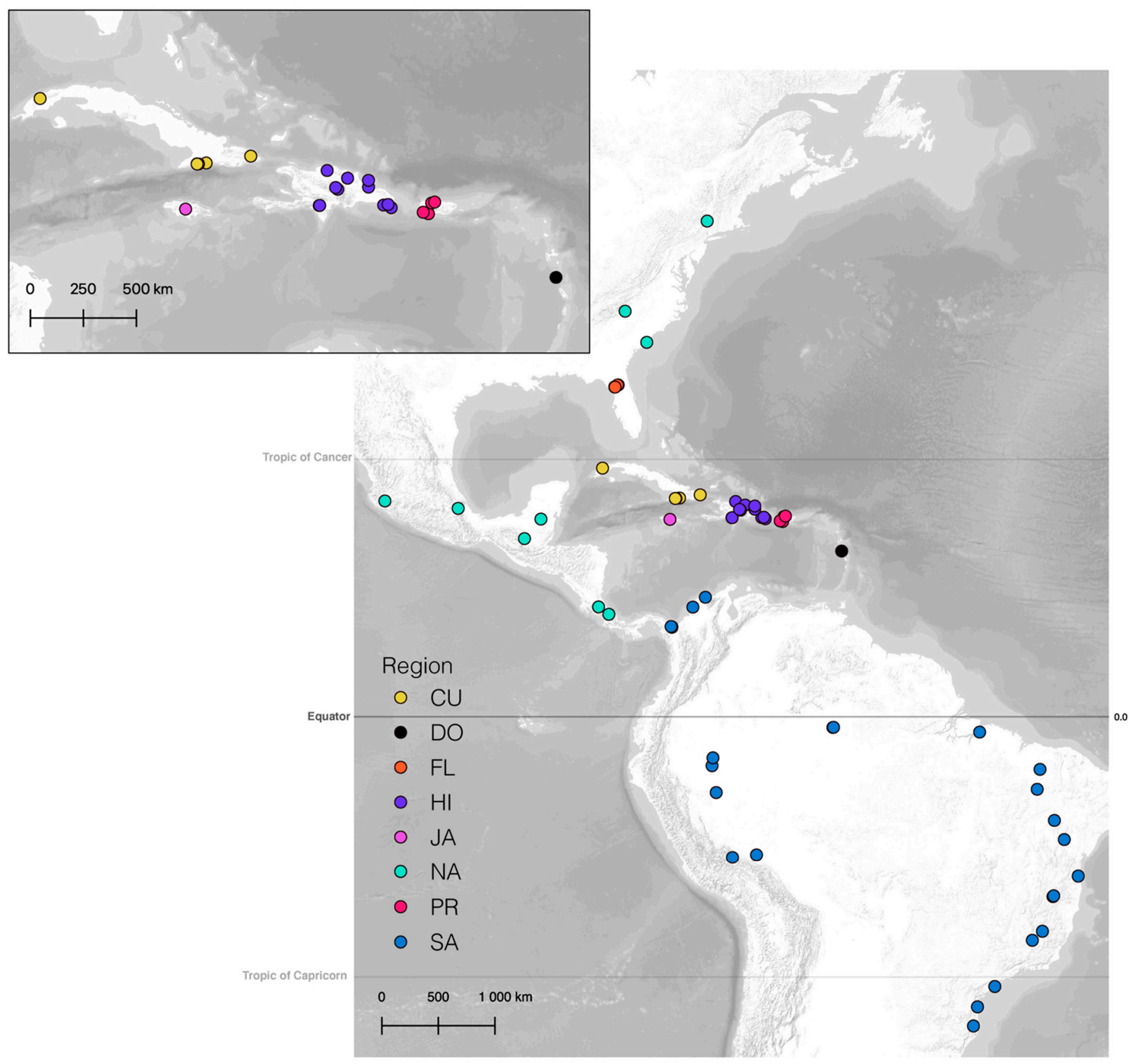

Figure 1. Map of collection localities of all specimens included in analysis. Points are colored by biogeographic area assigned for BioGeoBEARS analysis (see supporting material).

\subsection{Tissue Extraction and PCR}

Tissue samples were taken from the right legs, and DNA was isolated using the QIAGEN DNeasy Tissue Kit (Qiagen, Inc., Valencia, CA, USA). Fragments of one mitochondrial locus (CO1: cytochrome c oxidase subunit 1) and one nuclear locus (ITS-2: internal transcribed spacer 2) were sequenced. The $16 \mathrm{~S}$ data, along with the previous ITS-2 and CO1 data, were retrieved from McHugh et al. [51]. Both ITS-2 and CO1 have demonstrated utility in illuminating relationships between species-level and low-level taxonomic clades in previous arachnid phylogenetics studies $[59,60]$. The CO1 locus was amplified using the primers Jerry [61] and C1-N-2776 [62] for the majority of specimens $(n=43)$, while a select number were amplified using LCO1490 [63] and C1-N-2776 $(n=7)$, which resulted in a higher success rate of amplification within this group. The ITS2 locus was amplified using the primers ITS5.8S and ITS4S [64]. The conditions for each PCR are listed in Table 2. Sanger sequencing was conducted by the University of Vermont Cancer Center DNA Analysis Facility within 
the Vermont Integrative Genomics Resource (VIGR) facility. Additional sequences used to inform deficiencies in our South American Micrathena collection were retrieved from GenBank. All novel sequences have been submitted to GenBank (in progress).

Table 2. Polymerase chain reaction (PCR) conditions for ITS-2 and CO1. Conditions were split for $\mathrm{CO} 1$, given that two sets of primers were used.

\begin{tabular}{ccccc}
\hline \multicolumn{5}{c}{ Polymerase Chain Reaction (PCR) Conditions } \\
\hline Gene & Forward Primer & Reverse Primer & $\begin{array}{c}\text { Annealing } \\
\text { Temp. }\left({ }^{\circ} \text { C) }\right.\end{array}$ & $\begin{array}{c}\text { Fragment } \\
\text { Length (bp) }\end{array}$ \\
\hline $\begin{array}{c}\text { Internal } \\
\text { transcribed } \\
\text { spacer 2 (ITS-2) }\end{array}$ & ITS4 & ITS5.8 & 47 & $350-500$ \\
\hline $\begin{array}{c}\text { Cytochrome } \\
\text { oxidase subunit } \\
1 \text { (CO1) }\end{array}$ & JCO11490 & C1-N-2776 & 46 & $\sim 1250$ \\
\hline
\end{tabular}

\subsection{Alignment and Phylogeny Building}

Phred and Phrap $[65,66]$ were used to compile sequence chromatograms. Chromatograms were inspected and sequences were edited using the Chromaseq module [67] within the program Mesquite 3.61 [68]. Sequences were aligned using the MAFFT online service [69] with gaps treated as missing characters and all other settings set to default. The substitution models and partitioning schemes for a Bayesian analysis were selected with PartitionFinder 2.1 [70], using AIC (Akaike's information criterion) [71] amongst the 24 available models in MrBayes [72]. Sequence data were partitioned by gene, and additionally by codon, for CO1 as input for PartitionFinder. We ran a Bayesian inference using the CIPRES online portal [73] on a concatenated matrix where each locus was separately partitioned using MrBayes 3.2.7.a [72]. The Markov Chain Monte Carlo (MCMC) algorithm was run with four chains for 30,000,000 generations, sampling every 1000 generations. Tracer 1.71 [74] was used to verify the proper mixing of chains, to confirm that stationarity had been achieved, and to determine the adequate burn-in.

\subsection{Divergence Time Estimation and Biogeographic Modeling}

To estimate node ages among Micrathena, we used BEAST 2.60 [75] under a relaxed clock model. Because the South American species only had CO1 sequence data available, we used only this locus in the BEAST analysis. Terminal taxa were pruned for redundancy so that one representative of each critical species remained. BEAST analyses for CO1 were run with both an alignment partitioned by codon, using the best-fit models extracted from PartitionFinder [70] (GTR $+\mathrm{I}+\Gamma$ for position 1 , TVM $+\mathrm{I}+\Gamma$ for position 2 , and TRN $+\Gamma$ for position 3), along with an unpartitioned analysis, which was run using the best-fit model for $\mathrm{CO} 1$ overall $(\mathrm{GTR}+\mathrm{I}+\Gamma)$. Both analyses returned identical results. The analyses in BEAST were run for 30,000,000 generations, sampling every 1000 generations with a Yule Tree prior. Micrathena, along with closely related lineages, lack a fossil record, so the phylogeny was calibrated using the estimated age of Araneidae and the most recent common ancestor (MRCA), including Theridiidae and Araneidae derived from a recent fossil calibrated study by Kuntner et al. [76]. The minimum age of Araneidae was set as a normal prior with a mean of 70 million years and a standard deviation of 3 . The minimum age of Theridiidae + Araneidae was also set as a normal prior with a mean of 100 million years and a standard deviation of 9; both prior distributions covered the $95 \%$ confidence intervals derived from Kuntner et al. [76]. Based on the estimated substitution rates of $\mathrm{CO} 1$ that have been found to be consistent across spider lineages [76,77], the mitochondrial substitution rate parameter (ucld.mean) mean value was set to 0.0112 and the s.d. was set to 0.001 . We confined the monophyly of Micrathena based on the results of our Bayesian analyses. Tracer 1.7 [74] was 
again utilized to visualize the results of our node age estimation analysis, to determine burn-in and to check for stationarity.

An ancestral range analysis was conducted using the BioGeoBEARS v.1.1.2 package in $R$ [78]. The maximum range was constrained to three areas, due to the widespread distribution of some focal taxa. In this analysis, we employed our CO1 dated phylogeny with terminals pruned to represent single species or genetically distinct single-island endemics based on our Bayesian tree. We defined seven geographic areas: North America (NA), South America (SA), Florida (FL), Cuba (CU), Hispaniola (HI), Jamaica (JA), and Puerto Rico (PR) (see Supplementary File S1). Mexico, and all of Central America north of Panama, were included as part of North America, given that the edge of the Maya Block in southern Mexico corresponds to the southernmost boundary of the North American Tectonic Plate and that the Chorotega and Chortís blocks of Central America were associated with North America as a geologic entity for our focal time period [79-81]. Florida was coded as a separate entity from North America, as the land was unavailable until about 5 mya [82].

We tested a GAARlandia model and a no-GAARlandia model (the distribution was explained by overwater dispersal) by applying probabilities to paleogeographical-based time slices coded on the emergence or submergence of the defined areas at a given period, following Chamberland et al. [46] and Tong et al. [31] (see Supplementary Material). GAARlandia was modeled as the connections between islands making up the Greater Antilles, along with their connection to South America from 35-30 mya [20,21]. We also modeled the geologic splits among the Greater Antillean islands in both the GAARlandia and noGAARlandia models, specifically the opening of the Mona Passage between Hispaniola and Puerto Rico at 23 mya, and the opening of the Windward Passage, separating Cuba and Hispaniola, at 15 mya [20]. In addition, we encoded for the fluctuating emergence of Jamaica at various periods, and on the timing of the appearance and distance of Central America to other landmasses within the region [20]. In BioGeoBEARS and within $R$, we applied the dispersal-extinction-cladogenesis (DEC) and DEC $+\mathrm{J}$ models, the latter of which accounts for founder-event speciation. It should be mentioned that the DEC $+\mathrm{J}$ model has been criticized as a poor explanator of geographic range evolution due to its parameterization of the speciation mode, as opposed to speciation rate [83]. Here, we tested DEC and DEC + J under the no-GAARlandia and GAARlandia models. The Akaike information criterion (AIC) [71] and relative likelihoods were used to assess model probabilities, given the data. We compared the likelihood scores obtained from each run to test for significance ( $\triangle \mathrm{AICc}$ of 2 was considered significant) [84].

\subsection{Specimen Photography}

Specimen photographs, depicting morphological variation between the populations or species, were taken using a Canon 5D camera with a $65 \mathrm{~mm}$ macro $5 \times$ zoom lens attached to the Visionary Digital BK laboratory system rig (Dun Inc., Palmyra, VA, USA). Specimens were placed in a dish filled with alcohol-based hand sanitizer (65\% ethanol), and covered with a thin film of $95 \%$ ethanol to in order to produce a clear image. Multiple image slices were stacked using the Helicon Focus [85] and were refined in Adobe Photoshop 22.1, where dust and other residues were removed from the background and the image was fine-tuned to adjust for contrast and sharpness. Scale measurements for each specimen were also added via Photoshop. Figures were generated and edited using Adobe Illustrator and exported as PDFs.

\section{Results}

\subsection{Sequence Alignment}

A total of 76 sequences were generated from the CO1 and ITS2 fragments of the Micrathena sample set $\left(n_{\mathrm{CO} 1}=50, n_{\text {ITS2 }}=26\right)$. These were combined with sequences retrieved from data generated by McHugh et al. [51] to form a combined dataset of 405 sequences $\left(n_{\mathrm{CO} 1}=164, n_{\mathrm{ITS} 2}=131, n_{16 \mathrm{~S}}=110\right)$, representing 189 individuals. The additional $24 \mathrm{CO} 1$ 
sequences, representing unaccounted-for species, were retrieved from GenBank. Alignment lengths were CO1-1162 bp, 16S-458 bp, and ITS2-554 bp for a total of 2174 base pairs.

\subsection{Phylogenetics}

Relationships based on the Bayesian inference were robustly supported, with posterior probability values of most nodes $>0.95$ (Figure 2). Relationships within Micrathena militaris showed considerably lower support than the other nodes along the tree, as did some of the other fine-scale relationships highlighted in this analysis (mostly individual specimens representing tree tips) (Figures 2-5). However, support for major clade divisions and deeprooted nodes remained consistently robust throughout the concatenated phylogeny (Figure 2).

Our results support the monophyly of Micrathena, but reject the monophyly of Caribbean Micrathena (Figures 2-5). All named Micrathena species were monophyletic. Caribbean taxa are distributed among three species groups, previously defined by Magalhães and Santos [53] (Figure 3). We identified Caribbean Micrathena to belong to the nominal militaris-group, including M. sexspinosa, M. militaris, M. sagittata, and M. banksi (Figure 3). In addition, we substantiated the furcula-group, containing $M$. cubana and $M$. similis.

The gracilis-group, including $M$. gracilis and $M$. horrida, was additionally delineated but did not include $M$. forcipata in our multillocus analysis (Figure 3). Instead, we found that Micrathena forcipata was located as a sister to M. schreibersi, together forming the sister group to the furcula group. However, the topology of our CO1 trees indicated that the positionality of the furcula group (M. cubana and $M$. similis) and $M$. schreibersi were unstable. In our CO1 analysis, $M$. schreibersi is sister to the gracilis-group, instead of $M$. forcipata, while both $M$. schreibersi and the gracilis-group were, together, sisters to M. forcipata (Figure 4).

Our analysis also produced evidence in support of single-island endemism and island monophyly of Micrathena forcipata. High levels of island genetic structuring and relatively deep divergences were observed between $M$. forcipata from Cuba and $M$. forcipata from Hispaniola (Figures 2-5). At a finer scale, M. forcipata groups from Hispaniola further demonstrated intra-island structuring (Figure 2).

A Puerto Rican M. militaris clade was nested within Hispaniolan M. militaris; thus, it is not a single-island endemic (Figure 2). Micrathena horrida from Cuba, Jamaica, and Central America were not found to be genetically distinct from one another, but were distinct from South American M. horrida (Figures 2-5). Furthermore, M. sagittata from Mexico, North America (South Carolina), and Florida were genetically distinct from one another, and may represent isolated, morphologically similar, but distinguishable species (Figures 2 and 3, L. Shapiro unpublished data). A putative new species, sister to M. nigrichelis, was additionally delineated, here denoted as M. sp. (Figure 2). In the Bayesian analysis two South American Micrathena: M. perfida and M. beta were used as outgroups, as they were found to be sister to the least inclusive clade containing Caribbean Micrathena (Figure 2). 


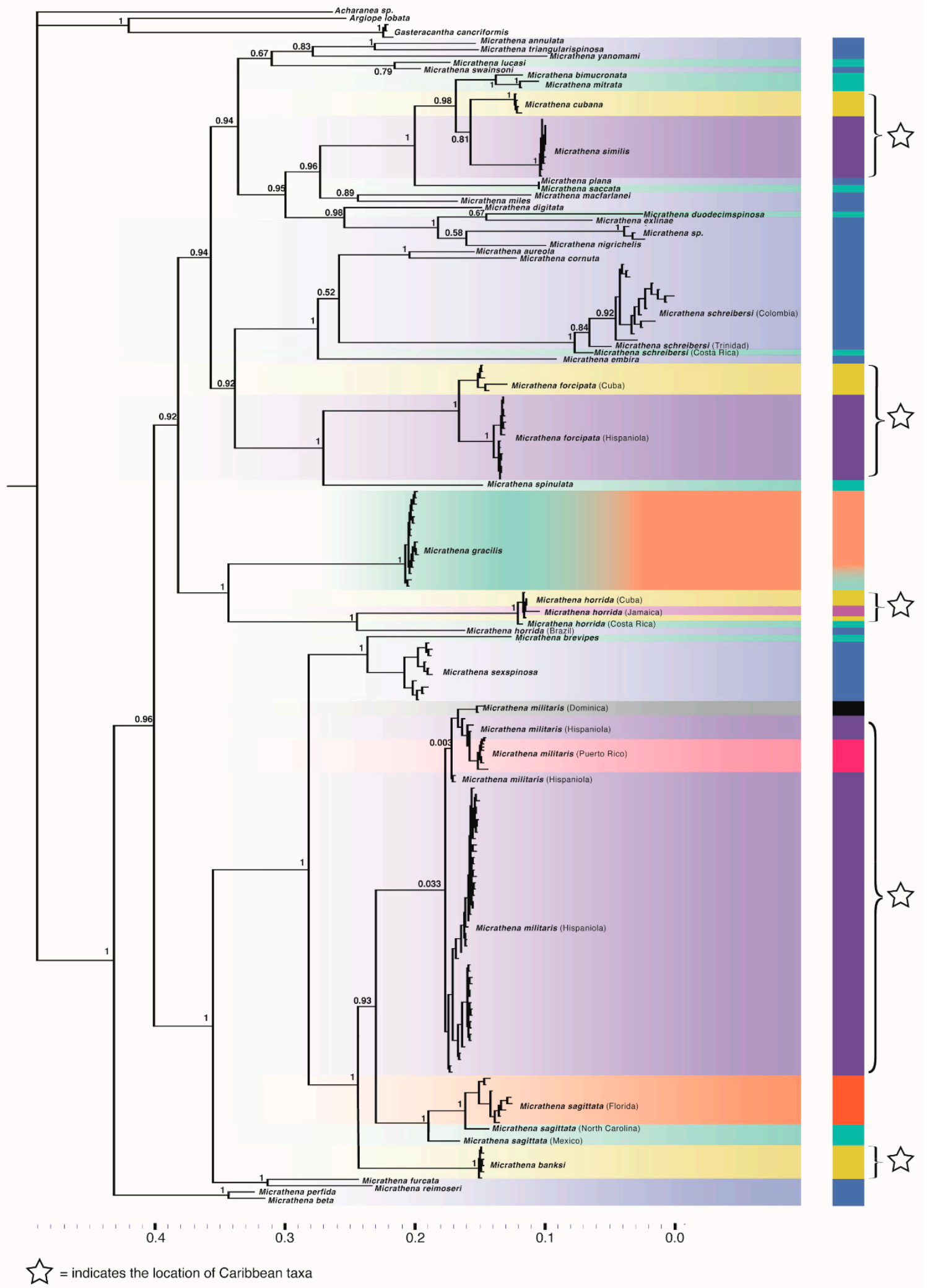

Figure 2. Complete consensus tree from MrBayes concatenated analysis depicting relationships among all sampled Micrathena species. Outgroups are located at the top of the phylogeny. Here, terminal individual labels have been replaced with species names along with locality. Overlaying colors are in accordance with color-coded map areas. M. gracilis was sampled from both North America and Florida and, therefore, is shaded with an analogous gradient. Stars represent the placement of Caribbean groups within the phylogeny. Posterior probability values are indicated. 


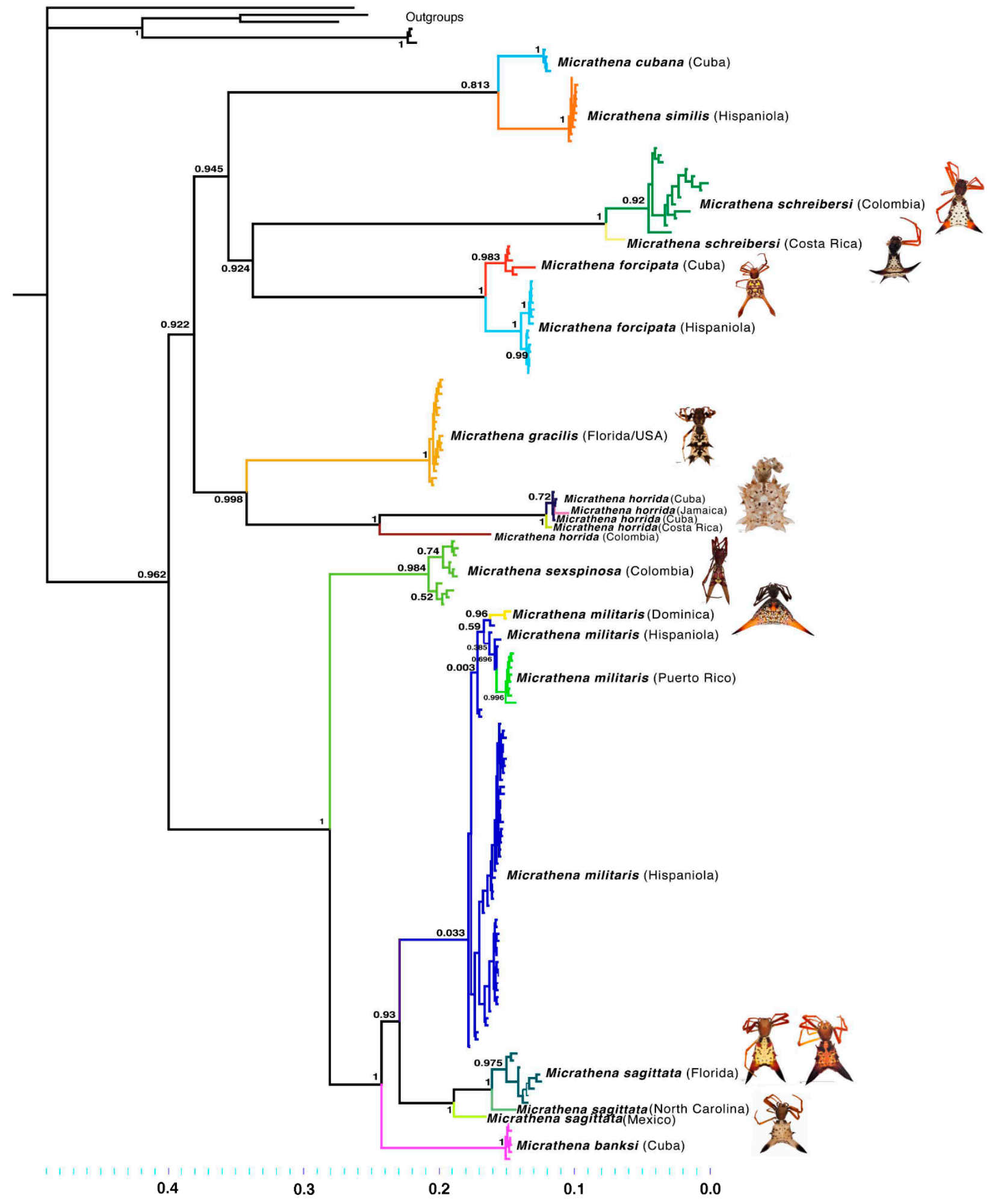

Figure 3. Pruned Bayesian inference tree depicting relationships among Caribbean species groups with associated posterior probability values. Branches are colored by species and individual taxa and have been replaced by species names at tips, but full clade structure is preserved. Micrathena dorsal habitus images represent adjacently located taxa. Branches are proportional to evolutionary distances. 


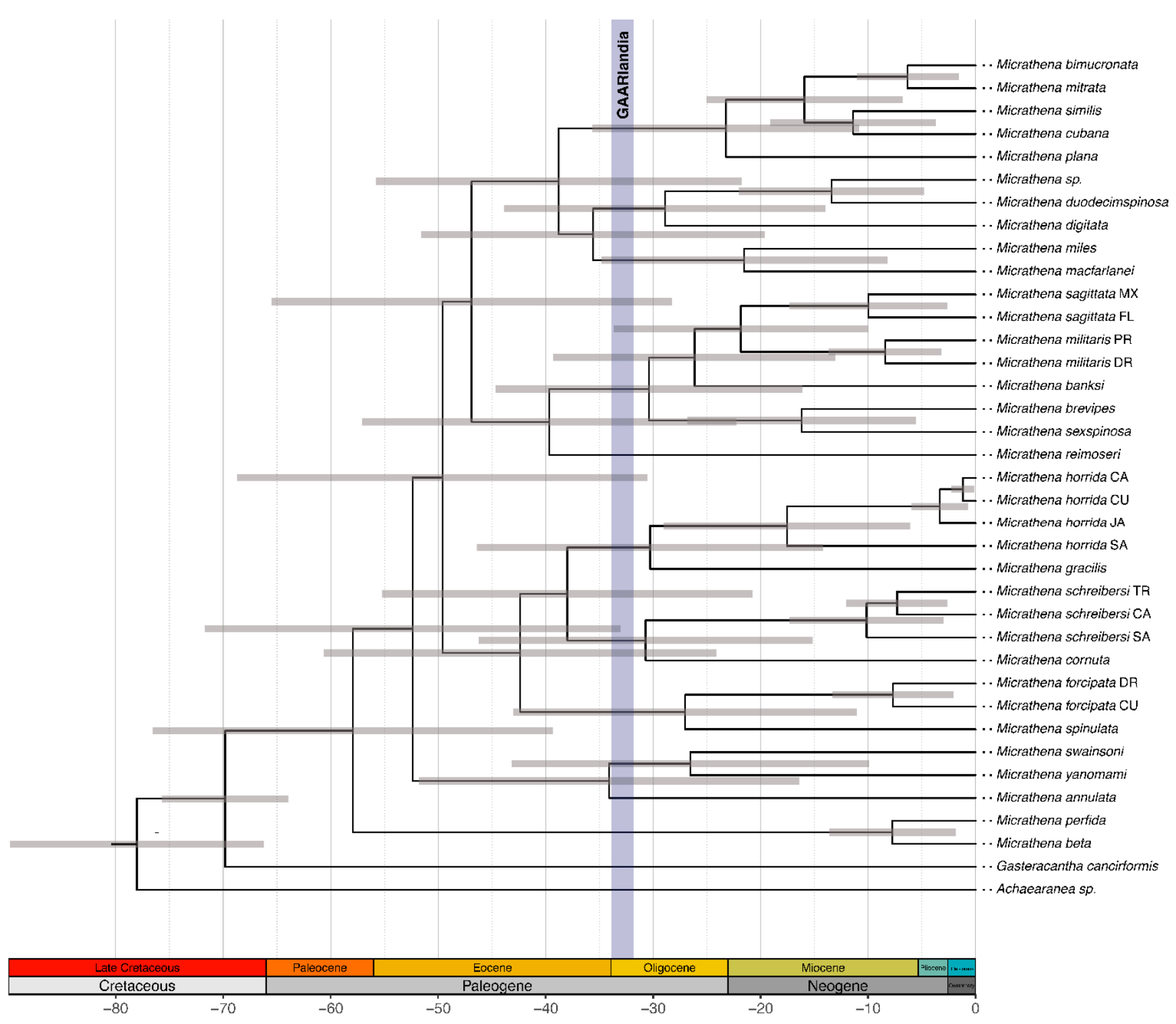

Figure 4. BEAST divergence time estimations of pruned taxa from CO1 data. Grey error bars show error margins around splits calculated in BEAST. Bottom scale is in millions of years and indicates associated geologic time units (periods on lower scale, epochs on upper scale). The timing of the GAARlandia landbridge is also shown from 33-35 Ma. Regional codes associated with taxon names are as follows: $\mathrm{CA}=$ Central America, $\mathrm{CU}=\mathrm{Cuba}, \mathrm{DR}=$ Dominican Republic, $\mathrm{FL}=$ Florida, JA = Jamaica, $\mathrm{MX}=$ Mexico, $\mathrm{PR}=$ Puerto Rico, $\mathrm{TR}=$ Trinidad . 
BioGeoBEARS DEC $+\mathrm{J}$ on Micrathena NO GAARlandia ancstates: global optim, 2 areas max. $d=0.0041 ; e=0.0011 ; j=0.2348 ; L n L=-81.87$

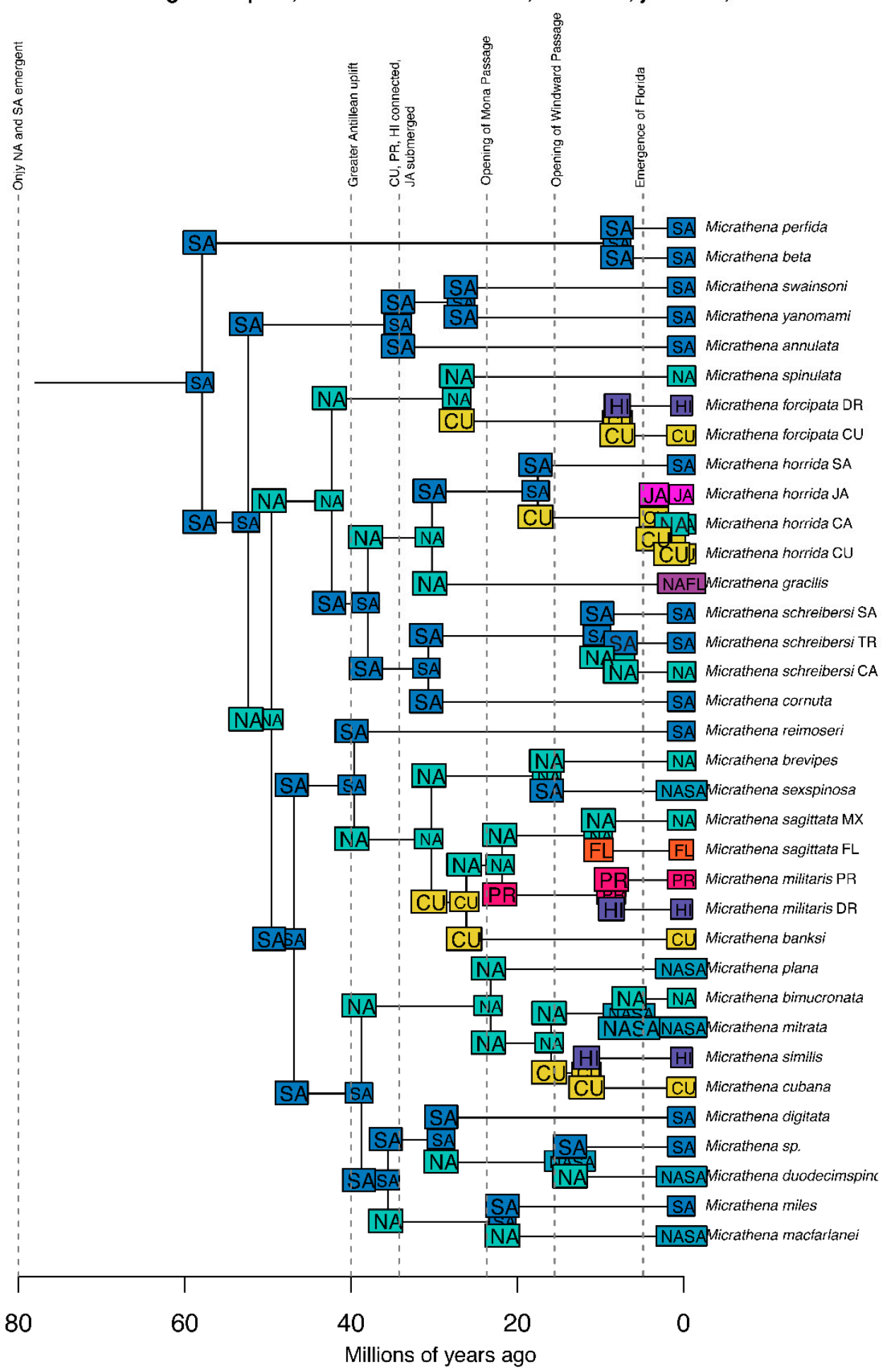

Figure 5. Ancestral range estimation output from BioGeoBEARS on the DEC + J no-GAARlandia model. Colored nodes indicate the most probable range of the MRCA (most recent common ancestor); $\mathrm{SA}=$ South America, NA = North America + Central America, CU = Cuba, PR = Puerto Rico, $\mathrm{HI}=$ Hispaniola, $\mathrm{FL}=$ Florida, $\mathrm{JA}=$ Jamaica. Some boxes indicate multiple probable ranges. Boxes are colored by species area labels (See Figure 1). Relevant geologic events corresponding with BioGeoBEARS time slice inputs (see Supplementary Material) are indicated by dotted lines. 


\subsection{Divergence Times}

Only CO1 data were used to build our dated phylogeny, as sequences were available for various South American taxa for which data on other loci were absent. BEAST analyses indicated that the age of Araneidae was estimated at 70 my (64-76), while the age of the Araneidae-Theridiidae split was placed at $78 \mathrm{my}$ (67-91) (Figure 4). The age of Micrathena was estimated to be around 58 my (33-71) (Paleocene, Thanetian, supported by Garrison et al. [86]), corroborating that they are representative of a relatively old New World araneid lineage and were present in the Caribbean region within the timing of the GAARlandia landbridge (Figure 4). Caribbean lineages diverged from mainland groups at variable geologic timepoints, with the oldest split dating back to around 30 mya between Cuba and North America and, additionally, implied five possible colonizations of the Caribbean (Figure 4). More recent Caribbean taxa, exemplified by M. cubana and M. similis, split from their Mexican and Central American relatives ( $M$. mitrata and $M$. bimucronata) at approximately 16 mya (Figure 4 ). The Caribbean and Central American lineages of M. horrida split from South American M. horrida at around 17 mya (Figure 4). Deep divergences between Mexican and Floridian M. sagittata were also suggested, with a split occurring approximately 10 mya (Figures 2-4). Caribbean Micrathena were ostensibly polyphyletic (Figures 2-5).

For further detail on topological comparisons between the Bayesian and CO1 BEAST trees, see Supplementary File S3.

\subsection{Biogeographic Patterns}

\subsubsection{Overview}

The ancestral range reconstruction in BioGeoBEARS suggested five independent colonizations of the Caribbean by Micrathena (the similis / cubana clade, banksi clade, militaris clade, horrida clade, and forcipata clade) (Figure 5). The larger banksi/militaris group is considered a Caribbean clade, but $M$. banksi and M. militaris from Hispaniola and Puerto Rico each arrived to the Greater Antilles independently (Figure 6). Micrathena originated in South America; an early branching South American lineage is sister to a lineage represented by another South American clade that is then, in turn, sister to the rest of the genus, including further South American members and those found in North and Central America and the Caribbean (Figure 5). There existed an early split between South and North American Micrathena 52 million years ago and, subsequently, multiple bifurcations between North/Central and South American Micrathena occurred thereafter (Figure 5). These results indicated that a fraction of Micrathena, other than the swainsoni and perfida clades, were indeed North American/Central American in origin, the ancestor having split from South America at this 52 mya timepoint, and this clade originating in North America 50 million years ago (Figure 5).

Four of the five clades containing Greater Antillean taxa are North American/Central American in origin (Figure 5). M. horrida is the exception, with South America denoted as ancestral, originating about $17 \mathrm{ma}$ (Figure 5). However the common ancestor of $M$. horrida and M. gracilis appears to be North American (30 Ma) (Figure 5). While Cuba is resolved as ancestral to the entirety of the sagitatta/militaris clade (including M. banksi), North America is the origin of M. militaris from both Puerto Rico and Hispaniola (its pre-dispersal to Puerto Rico was approximately $21 \mathrm{ma}$ ) (Figure 5). After colonization from South America, M. horrida appears to have diversified to form the Central American, Jamaican, and Cuban clades. Jamaican M. horrida split off from this group first at 3.3 Ma, with North/Central American $M$. horrida and Cuban M. horrida subsequently bifurcating at $1.18 \mathrm{Ma}$ (Figure 5). 

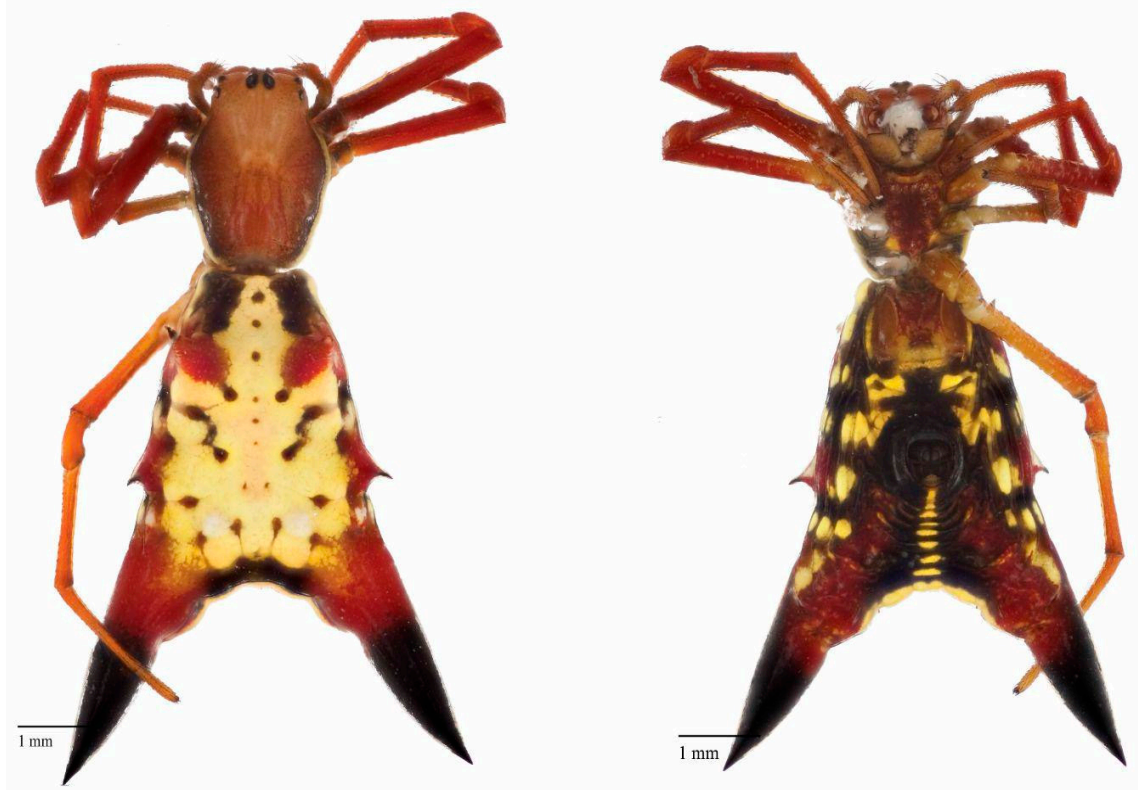

\section{M. sagittata (Florida)}
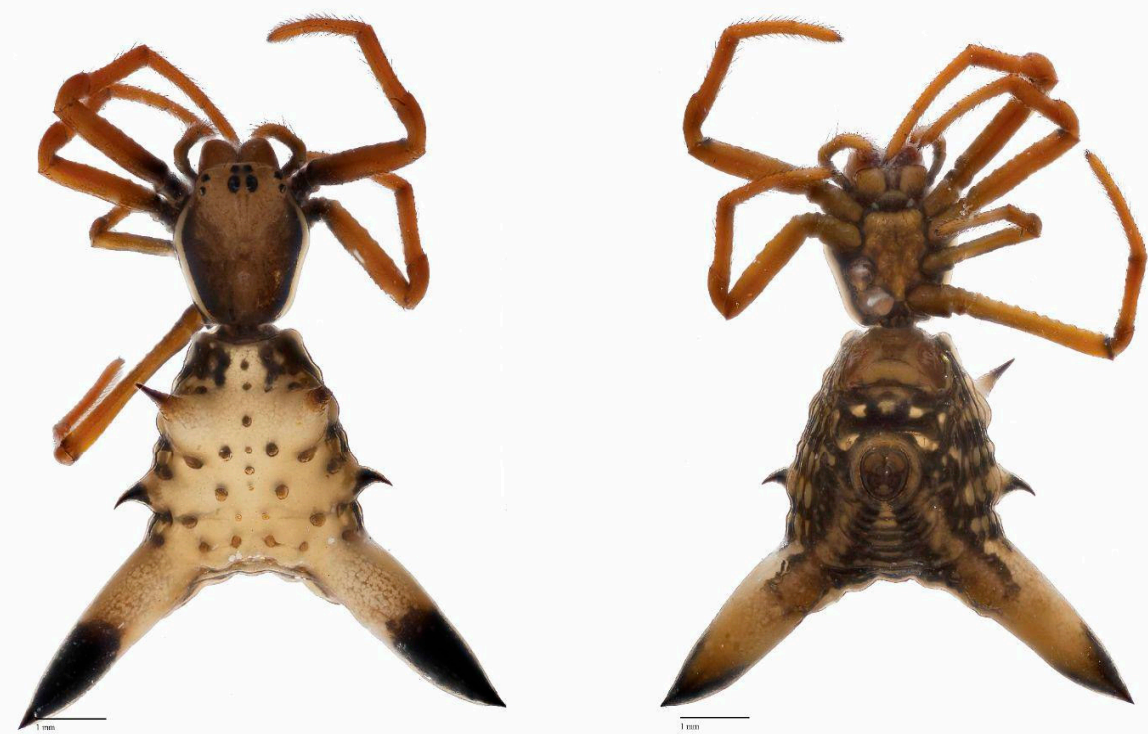

\section{M. sagittata (Mexico)}

Figure 6. High-resolution composite photographs of female M. sagittata specimens from Florida and Mexico depicting morphological variation between populations. Images are of dorsal and ventral habitus of each specimen. Scale bars are associated with each photograph (all lines are $1 \mathrm{~mm}$ in length). Habitus shape, along with posterior spine proportion and form, differ between the two groups, although spine number is consistent. Posterior spines of M. sagittata from Mexico appear more rounded and wider-set than Floridian $M$. sagittata. Obvious differences in coloration are apparent, with Mexican M. sagittata lacking the bright red and yellow pigmentation of Floridian M. sagittata on dorsal and ventral sides. Further sampling of Mexican M. sagittata is necessary to ensure within-population morphology is consistently distinct from Floridian M. sagittata.

Cuba was the first of the Greater Antillean islands to be colonized by South and North/Central American ancestors among all Caribbean groups in our analyses, preceding dispersal to other Caribbean islands (Puerto Rico, Hispaniola, or Jamaica (or mainland sources 
in select aforementioned cases)) (Figure 5). The initial splits between mainland and Cuban taxa occur at $27 \mathrm{Ma}$ (in the M. spinulata/M. forcipata group), $17 \mathrm{Ma}$ (amongst M. horrida), $30 \mathrm{Ma}$ (in the M. militaris clade), and $16 \mathrm{Ma}$ (within the M. simils/M.cubana/M. mitrata clade) (Figure 5).

We additionally observed multiple inter-island colonization events within the Greater Antilles; this included movement from Puerto Rico to Hispaniola at 8 mya within M. militaris, and two Cuba-Hispaniola splits at 7 and 11 mya within $M$. forcipata and between $M$. cubana and $M$ similis (Figure 5).

\subsubsection{Vicariance vs. Long Distance Dispersal}

The DEC + J no-GAARlandia hypothesis demonstrated the best statistical fit, given our input phylogeny, applied time-slices, and affiliated chrono-geographical probabilities (Table 3). The model comparison using AICc also distinguished the BAYAREALIKE $+\mathrm{J}$ as significant (Table 3). The top three models determined by AICc were all representative of no-GAARlandia hypotheses (Table 3) with mixed support for lower-ranked models, although none are of statistical significance (Table 3). Both the model ranking and BioGeoBEARS results are in agreement that colonization events are not tied to dispersal via the GAARlandia landbridge.

Table 3. BioGeoBEARS model probabilities and rankings. Six models were used in our analysis (DEC, DEC + J, BAYAREALIKE, BAYAREALIKE + J, DIVALIKE, DIVALIKE + J) to test data in the presence or absence of GAARlandia (GAARlandia and no-GAARlandia models). $L n L$ is log likelihood, $d$ is dispersal rate, $e$ is extinction rate, $j$ is the relative probability of founder event speciation at cladogenesis, AICc is Akaike's information criterion (with correction for smaller sample sizes), AICc weight is the normalized relative model likelihood, and $\triangle A I C c$ is AIC - $\min ($ AIC).

\begin{tabular}{|c|c|c|c|c|c|c|c|c|}
\hline Model & $\operatorname{LnL}$ & $\begin{array}{c}\text { Number } \\
\text { of Parameters }\end{array}$ & $d$ & $e$ & $j$ & $A I C c$ & AICc Weight & $\triangle A I C c$ \\
\hline $\begin{array}{c}\text { DEC + J } \\
\text { no-GAARlandia }\end{array}$ & -81.87 & 3 & 0.0041 & 0.0011 & 0.2 & 170.5 & 0.56 & 0 \\
\hline $\begin{array}{c}\text { BAYAREALIKE + J } \\
\text { no-GAARlandia }\end{array}$ & -82.46 & 3 & 0.0019 & 0.01 & 0.2 & 171.7 & 0.31 & 1.2 \\
\hline $\begin{array}{c}\text { DIVALIKE + J } \\
\text { no-GAARlandia }\end{array}$ & -83.53 & 3 & 0.0048 & 0.001 & 0.2 & 173.8 & 0.11 & 3.3 \\
\hline $\begin{array}{l}\text { BAYAREALIKE + J } \\
\text { GAARlandia }\end{array}$ & -85.26 & 3 & 0.023 & 0.011 & 0.8 & 177.3 & 0.019 & 6.8 \\
\hline $\begin{array}{c}\text { DIVALIKE } \\
\text { no-GAARlandia }\end{array}$ & -95.23 & 2 & 0.013 & 0.0033 & 0 & 194.8 & $2.9 \times 10^{-6}$ & 24.3 \\
\hline $\begin{array}{c}\text { DEC + J } \\
\text { GAARlandia }\end{array}$ & -94.48 & 3 & 0.025 & $1.00 \times 10^{-12}$ & 2.4 & 195.7 & $1.90 \times 10^{-6}$ & 25.2 \\
\hline $\begin{array}{l}\text { DIVALIKE + J } \\
\text { GAARlandia }\end{array}$ & -97.42 & 3 & 0.027 & $1.00 \times 10^{-12}$ & 1.7 & 201.6 & $9.90 \times 10^{-8}$ & 31.1 \\
\hline $\begin{array}{c}\text { DEC } \\
\text { no-GAARlandia }\end{array}$ & -99.69 & 2 & 0.013 & 0.0063 & 0 & 203.8 & $3.40 \times 10^{-8}$ & 33.3 \\
\hline $\begin{array}{l}\text { BAYAREALIKE } \\
\text { no-GAARlandia }\end{array}$ & -107.9 & 2 & 0.017 & 0.025 & 0 & 220.2 & $8.90 \times 10^{-12}$ & 49.7 \\
\hline $\begin{array}{l}\text { BAYAREALIKE } \\
\text { GAARlandia }\end{array}$ & -112 & 2 & 0.24 & 0.025 & 0 & 228.4 & $1.50 \times 10^{-13}$ & 57.9 \\
\hline $\begin{array}{l}\text { DIVALIKE } \\
\text { GAARlandia }\end{array}$ & -112.8 & 2 & 0.11 & 0.0058 & 0 & 230 & $6.90 \times 10^{-14}$ & 59.5 \\
\hline DEC GAARlandia & -112.9 & 2 & 0.16 & 0.01 & 0 & 230.2 & $6.00 \times 10^{-14}$ & 59.7 \\
\hline
\end{tabular}




\section{Discussion}

Molecular analyses, with the expanded taxon sampling of Micrathena, resolved the genus as monophyletic with polyphyletic Caribbean taxa (Figures 2-5), consistent with the findings of McHugh et al. [51], Crews and Esposito [36], and Magalhães and Santos [53] (Figures 2-5). We detected five independent colonization events to the Caribbean from varying mainland sources (Figure 5). While South America was the ancestral Micrathena range, four of the five Caribbean groups were actually North American/Central American in origin (Figure 5), corroborating evidence by other authors [36]. Crews and Esposito [36] found evidence that Micrathena had repeatedly dispersed to the Caribbean (six times) and suggested that GAARlandia likely played some role in this dispersal. We did not find evidence for the latter hypothesis [36,51]. Rather, the BioGeoBEARS results and the biogeographic model ranking indicated that Micrathena colonized the Caribbean multiple times, but each time outside of the timespan of the proposed GAARlandia landbridge.

In addition to the dispersal from continental sources, we found evidence for movement among islands, as well as the reverse colonization of North America from Cuba (Figure 5). The phenomenon of movement from island-to-continent has been documented in other spider lineages, including Deinopis [46] and Tetragnatha [87], adding to the growing frequency of this pattern observed in arachnids, even across groups with variable dispersal strategies [87]. Movement among the Greater Antillean islands reflected both long-distance dispersal and the dispersal to nearby islands (e.g., two pairs of HI-CU sister taxa and the M. militaris groups from PR and HI) (Figures 2-5).

Independent dispersals at various geologic timepoints (Figure 5) suggested that stochastic events, such as extreme weather events (e.g., hurricanes) or ocean currents, could have played a role in transporting Micrathena across the Caribbean, as proposed for other arthropod groups [88-90]. Given that the Caribbean lineages of Micrathena have a North/Central American origin, the loop current, wrapping around the Gulf of Mexico, entering by the Yucatán peninsula, and exiting via the straights of Florida [91], may be of particular import as it brushes close to Greater Antillean islands. The long-distance dispersal, via rafting in arachnids, has been documented in Moggridgea mygalomorphs in Australia [92] and in Amaurobioides [93]. Paleocurrent directionality in the Caribbean, which most likely mirrors that of the Holocene (although a thruway between the Atlantic and Pacific existed before the closure of the Panama isthmus at 3.5 Ma) [94-96], and it can be hypothesized that the dispersal routes that allowed Micrathena to colonize the Caribbean reflect modern and paleooceanographic dynamics. Future investigations may consider integrating paleowind and paleocurrent data to better explain fine-scale dispersal routes of Caribbean colonization that criss-cross the region. While such analyses have been undertaken for Caribbean mammals in terms of utilizing "floating islands" [97], these data have not been applied to biogeographic investigations of spiders. However, hurricanes (with modern directionality) have been shown to be a mechanism important in arthropod dispersal [90] and the dispersal effects have also been empirically noted [89]. The habitat choice in Micrathena, often occupying the center of wide-open spaces in forests where the web and animal are readily exposed to weather conditions reaching inside the forest, could render them relatively prone to weather-related involuntary aerial dispersal.

This study adds to the growing composite of data suggesting manifold Caribbean dispersals in Micrathena and indicates that, although they are considered relatively poor dispersers due to their apparent bulkiness and elaborate spine coverage, Micrathena may actually be relatively proficient dispersers. We would predict this dispersal would mostly occur as juveniles, when they are less heavily ornamented. Other large araneids, including Nephila [98] and various Argiope and Araneus species, do balloon [56]. Not much is known about the physical capacity for dispersal in Micrathena, and biogeographic investigations may benefit from increased physiological and behavioral analyses of the genus.

We recovered four distinct Micrathena clades containing Caribbean taxa, which roughly correspond to the species-groups defined by Magalhães and Santos [53] and are corroborated by McHugh et al. [51]: the militaris-group, the gracilis-group, and the furcula-group + M. forcipata 
(Figure 3, Table 4). Like McHugh et al. [51], our analyses do not place M. forcipata within the gracilis group. However, the placement of $M$. forcipata differs from McHugh et al. [51] and is influenced by taxon sampling and phylogenetic methods (Table 4). It is likely that gaps in taxon sampling are responsible for the instability of $M$. schreibersi and the furcula group, that is noted between the multilocus and the $\mathrm{CO} 1$ analyses.

Table 4. Comparisons between species-group delineations for three Micrathena phylogenetic analyses performed by Magalhaēs et al. [53], McHugh et al. [51], and this investigation (multilocus datset, Figures 1 and 2). Caribbean species groups are listed along with species belonging to that group in each study. Additional notes on the differing position of M. schreibersi, as it relates to these groups, the study by McHugh et al. [51], and this analysis, are listed as footnotes.

\begin{tabular}{cccc}
\hline Species-Group & Magalhaēs et al., 2012 & McHugh et al., 2014 & $\begin{array}{c}\text { Current } \\
\text { Micrathena Study }\end{array}$ \\
\hline furcula & M. cubana, M. similis & M. cubana, M. similis & M. cubana, M. similis \\
\hline \multirow{2}{*}{ militaris } & M. banksi, M. militaris, M. & M. banksi, M. militaris, & M. banksi, M. militaris, \\
& sagittata, M. sexspinosa & M. sagittata, & M. sagittata, \\
gracilis & M. horrida, M. gracilis, & M. horrida, & M. sexspinosa \\
\hline M. forcipata & M. gracilis ${ }^{1}$ & M. horrida, \\
& M. gracilis ${ }^{2}$ \\
\hline
\end{tabular}

${ }^{1}$ M. schreibersi is the sister to the gracilis group; M. forcipata is the sister to the furcula group. ${ }^{2}$ M. schreibersi is the sister to M. forcipata, and both are sisters to the furcula group.

Our analyses indicated deep divergences within 'widespread taxa', suggesting that such taxa would be better characterized as multiple single-island endemics. For example, M. forcipata from Cuba and Hispaniola are genetically distinct from one another, as indicated by deep branching separating the two on the phylogeny. These taxa may also be distinguishable based on morphology (Figure 3 and L. Shapiro's unpublished data). The divergence among these similar taxa is likely due to the segregation of these two islands by the Windward Passage, acting as a geographic barrier post-dispersal (Figures 2-5). While McHugh et al. [51] also determined that the $M$. militaris groups represent single-island endemics from Puerto Rico and Hispaniola, we found that, although M. militaris from Puerto Rico are monophyletic, they are nested within the Hispaniolan members of the species, hence rejecting a model of purely single-island endemics in this genus (Figure 2).

Genetic divergences between M. sagittata from North America (North Carolina), Florida, and Mexico were also noted in our analyses, where the Mexican M. sagittata is the sister to the North American group (Figures 2 and 3). Morphological distinctions between Mexican M. sagittata, in comparison to our M. sagittata sample from Florida, can be clearly observed (Figure 6). An additional putative, currently undescribed sister species to $M$. nigrichelis was identified in the phylogeny, Micrathena sp. The preliminary habitus photographs of $M$. sp. are displayed in Figure 7. Integrative genetic and morphological analyses are currently underway to solidify evidence for the species delimitations of new clades and divergent species uncovered in this study.

Our work, combined with previous biogeographic analyses, substantiates Micrathena spiders as an excellent model for Caribbean biogeography of a dispersal-prone lineage. The additional depth in taxon sampling of Micrathena and the related genera, especially across Central and South America, as well as expanded data with next-generation sequencing and the greater availability of fossil evidence for calibration, will add to the resolution of factors influencing biodiversity in this region. 

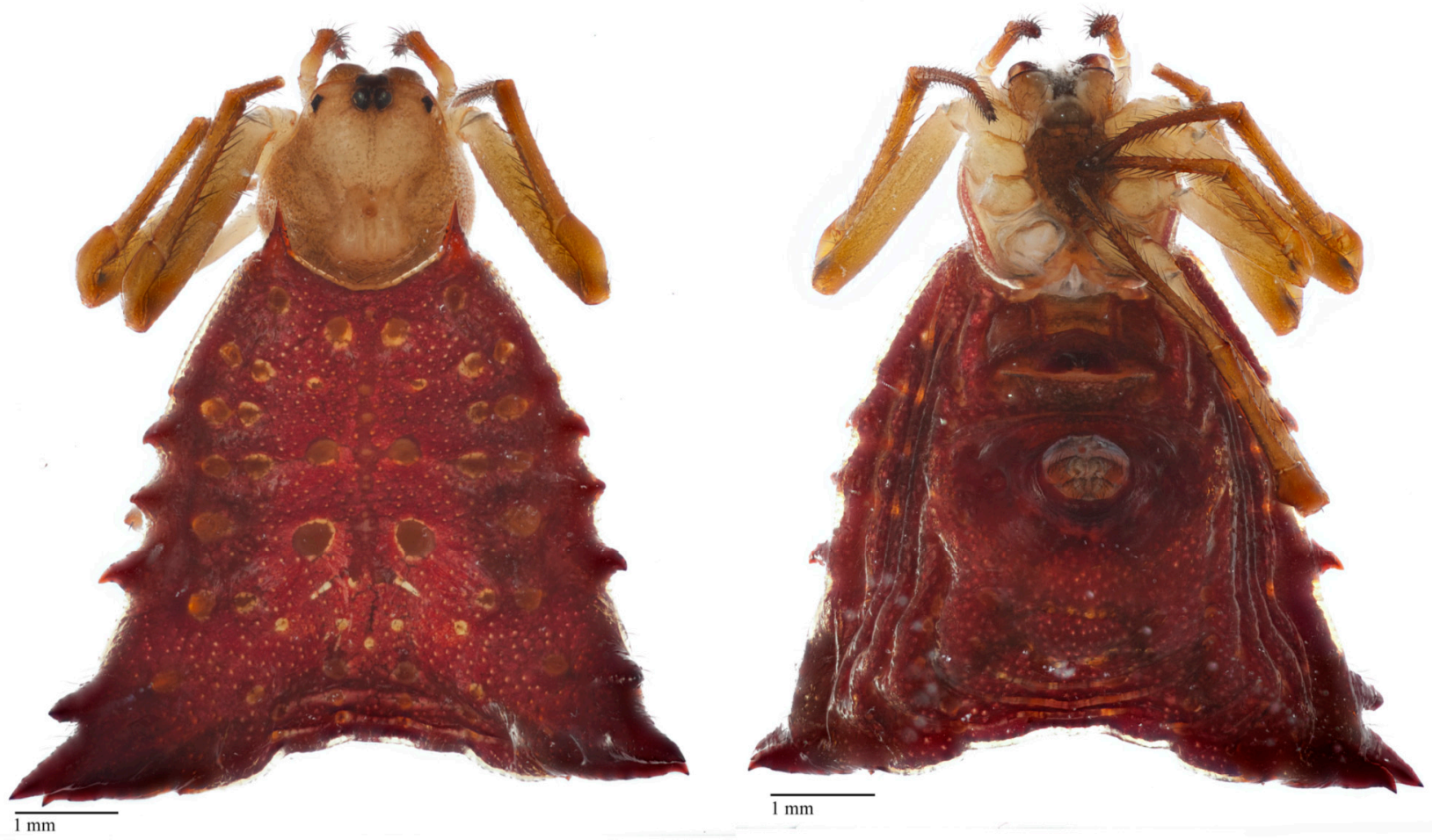

Figure 7. High-resolution composite photographs of putative new species $M$. sp. from Colombia. Photographs depict dorsal and ventral habitus of a female specimen. Future studies will hopefully provide more data detailing important morphological characters. Scale is depicted at the bottom of each photograph.

\section{Conclusions}

We present a detailed molecular phylogenetic and biogeographic analysis of Micrathena, demonstrating that the group likely colonized the Caribbean region multiple times independently during the last 30 million years, and that diversification was likely a result of multiple overwater dispersal events and not GAARlandia vicariance. This finding suggests that Micrathena, while potentially dispersal-limited due to its size and morphology, have nevertheless been carried across oceanic barriers to colonize Caribbean islands five times in 30 million years, perhaps as juveniles. We found interesting evidence for single-island endemics in M. forcipata and have unveiled the cryptic diversity in M. sagittata and within the genus altogether. Further studies will focus on taxonomic examinations of potential species uncovered in this phylogeny.

Supplementary Materials: The following are available online at https: / www.mdpi.com/article/ 10.3390/d14020128/s1, File S1: Dispersal probabilities and geography input for BioGeoBEARS, File S2: List of Micrathena species in study, File S3: Comparison of concatenated Bayesian and BEAST phylogenies, File S4: Raw BEAST.xml output file.

Author Contributions: Conceptualization, L.S. and I.A.; methodology L.S. and I.A.; software, L.S. and I.A.; formal analysis, L.S. and I.A.; investigation, L.S. and I.A.; resources, I.A.; data curation, L.S. and I.A.; writing—original draft preparation, L.S.; writing—review and editing, L.S., I.A. and G.J.B.; visualization, L.S. and I.A.; supervision, I.A. and G.J.B.; project administration, I.A.; funding acquisition, I.A. and G.J.B. All authors have read and agreed to the published version of the manuscript.

Funding: This research was funded by the National Science Foundation, grants numbered DEB1314749 and DEB-1050253 awarded to G. Binford and I. Agnarsson, and by a grant from the National Geographic Society (WW-203R017) to I. Agnarsson.

Institutional Review Board Statement: All material was collected under appropriate collection permits and approved guidelines. 
Informed Consent Statement: Not applicable.

Data Availability Statement: Code can be found at https://github.com/lkshapir/Micrathena paper_scripts (accessed on 6 October 2021).

Acknowledgments: We would like to thank all members of the CarBio team who were involved in collecting and cataloguing specimens used in this study. We thank members of the Agnarsson laboratory-specifically Lisa Chamberland and Laura Caicedo-Quiroga for their guidance and advice in developing this project, and Matjaz Gregoric and Ren-Chun Cheng of the Kuntner lab in Slovenia for providing outgroup sequence data on Argiope. Special thanks to Anne McHugh who initiated this research project and published a paper on earlier findings.

Conflicts of Interest: The authors declare no conflict of interest.

\section{References}

1. Wilson, E.O.; MacArthur, R.H. The Theory of Island Biogeography; Princeton University Press: Princeston, NJ, USA, 1967.

2. Carlquist, S.J. Island Biology; Columbia University Press: New York, NY, USA, 1974.

3. Whittaker, R.J. Island Biogeography; Oxford University Press: Oxford, UK, 1998.

4. Whittaker, R.J.; Fernández-Palacios, J.M. Island Biogeography: Ecology, Evolution, and Conservation; Oxford University Press: Oxford, UK, 2007.

5. Gillespie, R. Community Assembly Through Adaptive Radiation in Hawaiian Spiders. Science 2004, 303, 356-359. [CrossRef]

6. Craig, D.A.; Currie, D.C.; Joy, D.A. Geographical history of the central-western Pacific black fly subgenus Inseliellum (Diptera: Simuliidae: Simulium) based on a reconstructed phylogeny of the species, hot-spot archipelagoes and hydrological considerations. J. Biogeogr. 2001, 28, 1101-1127. [CrossRef]

7. Ricklefs, R.; Bermingham, E. The West Indies as a laboratory of biogeography and evolution. Philos. Trans. R. Soc. B Biol. Sci. 2008, 363, 2393-2413. [CrossRef]

8. Myers, N.; Mittermeler, R.A.; Mittermeler, C.G.; Da Fonseca, G.A.B.; Kent, J. Biodiversity hotspots for conservation priorities. Nature 2000, 403, 853-858. [CrossRef] [PubMed]

9. Rosen, D.E. A Vicariance Model of Caribbean Biogeography. Syst. Biol. 1975, 24, 431-464. [CrossRef]

10. Pindell, J.L.; Barrett, S.F. Geologic evolution of the Caribbean region: A plate-tectonic persepective. In Decade of American Geology; Case, J.E., Dengo, G., Eds.; Geological Society of America: Boulder, CO, USA, 1990; The Geology of North America v. H; pp. 405-432.

11. Pindell, J.L. Evolution of the Gulf of Mexico and the Caribbean. In Caribbean Geology, an Introduction; Donovan, S.K., Jackson, T.A., Eds.; The University of West Indies Publishers' Association: Kingston, Jamaica, 1994; pp. 13-39.

12. Burke, K. Tectonic evolution of the Caribbean. Annu. Rev. Earth Planet. Sci. 1988, 16, 201-230. [CrossRef]

13. Donnelly, T.W. Geologic constraints on Caribbean biogeography. In Zoogeography of Caribbean Insects; Liebherr, J.K., Ed.; Cornell University Press: Ithaca, NY, USA, 1988; pp. 15-37.

14. Alonso, R.; Crawford, A.J.; Bermingham, E. Molecular phylogeny of an endemic radiation of Cuban toads (Bufonidae: Peltophryne) based on mitochondrial and nuclear genes. J. Biogeogr. 2012, 39, 434-451. [CrossRef]

15. Keppel, G.; Lowe, A.J.; Possingham, H.P. Changing Perspectives on the Biogeography of the Tropical South Pacific: Influences of Dispersal, Vicariance and Extinction. J. Biogeogr. 2009, 36, 1035-1054. [CrossRef]

16. Crews, S.C.; Gillespie, R.G. Molecular systematics of Selenops spiders (Araneae: Selenopidae) from North and Central America: Implications for Caribbean biogeography. Biol. J. Linn. Soc. 2010, 101, 288-322. [CrossRef]

17. Heinicke, M.P.; Duellman, W.E.; Hedges, S.B. Major Caribbean and Central American frog faunas originated by ancient oceanic dispersal. Proc. Natl. Acad. Sci. USA 2007, 104, 10092-10097. [CrossRef]

18. Toussaint, E.F.A.; Balke, M. Historical biogeography of Polyura butterflies in the oriental Palaeotropics: Trans-archipelagic routes and South Pacific island hopping. J. Biogeogr. 2016, 43, 1560-1572. [CrossRef]

19. Agnarsson, I.; Cheng, R.-C.; Kuntner, M. A multi-clade test supports the intermediate dispersal model of biogeography. PLoS ONE 2014, 9, e86780. [CrossRef]

20. Iturralde-Vinent, M.A. Meso-Cenozoic Caribbean paleogeography: Implications for the historical biogeography of the region. Int. Geol. Rev. 2006, 48, 791-827. [CrossRef]

21. Iturralde-Vinent, M.A.; MacPhee, R.D.E. Paleogeography of the Caribbean region: Implications for Cenozoic biogeography. Bull. Am. Museum Nat. Hist. 1999, 238, 1-95.

22. Fabre, P.-H.; Vilstrup, J.T.; Raghavan, M.; Der Sarkissian, C.; Willerslev, E.; Douzery, E.J.P.; Orlando, L. Rodents of the Caribbean: Origin and diversification of hutias unravelled by next-generation museomics. Biol. Lett. 2014, 10, 20140266. [CrossRef] [PubMed]

23. Klemen, Č.; Ingi, A.; Binford, G.J.; Matjaž, K. Biogeography of the Caribbean Cyrtognatha spiders. Sci. Reports (Nature Publ. Group) 2019, 9, 1-14.

24. Esposito, L.A.; Bloom, T.; Caicedo-Quiroga, L.; Alicea-Serrano, A.M.; Sánchez-Ruíz, J.A.; May-Collado, L.J.; Binford, G.J.; Agnarsson, I. Islands within islands: Diversification of tailless whip spiders (Amblypygi, Phrynus) in Caribbean caves. Mol. Phylogenet. Evol. 2015, 93, 107-117. [CrossRef] 
25. Condamine, F.L.; Silva-Brandão, K.L.; Kergoat, G.J.; Sperling, F.A.H. Biogeographic and diversification patterns of Neotropical Troidini butterflies (Papilionidae) support a museum model of diversity dynamics for Amazonia. BMC Evol. Biol. 2012, 12, 82. [CrossRef]

26. Dziki, A.; Binford, G.J.; Coddington, J.A.; Agnarsson, I. Spintharus flavidus in the Caribbean-A 30 million year biogeographical history and radiation of a 'widespread species'. PeerJ 2015, 3, e1422. [CrossRef]

27. Pfingstl, T.; Lienhard, A.; Baumann, J.; Koblmüller, S. A taxonomist's nightmare-Cryptic diversity in Caribbean intertidal arthropods (Arachnida, Acari, Oribatida). Mol. Phylogenet. Evol. 2021, 163, 107240. [CrossRef]

28. Esposito, L.A.; Prendini, L. Island ancestors and New World biogeography: A case study from the scorpions (Buthidae: Centruroidinae). Sci. Rep. 2019, 9, 1-11. [CrossRef]

29. Chamberland, L.; Salgado-Roa, F.C.; Basco, A.; Crastz-Flores, A.; Binford, G.J.; Agnarsson, I. Phylogeography of the widespread Caribbean spiny orb weaver Gasteracantha cancriformis. PeerJ 2020, 8, e8976. [CrossRef]

30. Říčan, O.; Piálek, L.; Zardoya, R.; Doadrio, I.; Zrzavý, J. Biogeography of the Mesoamerican Cichlidae (Teleostei: Heroini): Colonization through the GAARlandia land bridge and early diversification. J. Biogeogr. 2013, 40, 579-593. [CrossRef]

31. Tong, Y.; Binford, G.; Rheims, C.A.; Kuntner, M.; Liu, J.; Agnarsson, I. Huntsmen of the Caribbean: Multiple tests of the GAARlandia hypothesis. Mol. Phylogenet. Evol. 2019, 130, 259-268. [CrossRef]

32. Agnarsson, I.; LeQuier, S.M.; Kuntner, M.; Cheng, R.-C.; Coddington, J.A.; Binford, G. Phylogeography of a good Caribbean disperser: Argiope argentata (Araneae, Araneidae) and a new 'cryptic'species from Cuba. Zookeys 2016, 25. [CrossRef] [PubMed]

33. Nieto-Blázquez, M.E.; Antonelli, A.; Roncal, J. Historical Biogeography of endemic seed plant genera in the Caribbean: Did GAAR landia play a role? Ecol. Evol. 2017, 7, 10158-10174. [CrossRef] [PubMed]

34. Dávalos, L.M. Phylogeny and biogeography of Caribbean mammals. Biol. J. Linn. Soc. 2004, 81, 373-394. [CrossRef]

35. Dávalos, L.M.; Turvey, S.T. West Indian mammals: The old, the new, and the recently extinct. In Bones, Clones, and Biomes: The History and Geography of Recent Neotropical Mammals; Patterson, B.D., Costa, L.P., Eds.; University of Chicago Press: Chicago, IL, USA, 2012; pp. 157-202.

36. Crews, S.C.; Esposito, L.A. Towards a synthesis of the Caribbean biogeography of terrestrial arthropods. BMC Evol. Biol. 2020, 20, 12. [CrossRef] [PubMed]

37. Philippon, M.; Cornée, J.-J.; Münch, P.; Van Hinsbergen, D.J.J.; Boudagher-Fadel, M.; Gailler, L.; Boschman, L.M.; Quillevere, F.; Montheil, L.; Gay, A.; et al. Eocene intra-plate shortening responsible for the rise of a faunal pathway in the northeastern Caribbean realm. PLOS ONE 2020, 15, e0241000.

38. Ali, J.R. Colonizing the Caribbean: Is the GAARlandia land-bridge hypothesis gaining a foothold? J. Biogeogr. 2012, 39, 431-433. [CrossRef]

39. Hedges, S.B. Paleogeography of the Antilles and Origin of West Indian Terrestrial Vertebrates. Ann. Missouri Bot. Gard. 2006, 93, 231-244. [CrossRef]

40. Ali, J.R.; Hedges, S.B. Colonizing the Caribbean: New geological data and an updated land-vertebrate colonization record challenge the GAARlandia land-bridge hypothesis. J. Biogeogr. 2021, 48, 2699-2707. [CrossRef]

41. Garrocq, C.; Lallemand, S.; Marcaillou, B.; Lebrun, J.; Padron, C.; Klingelhoefer, F.; Laigle, M.; Münch, P.; Gay, A.; Schenini, L.; et al. Genetic Relations Between the Aves Ridge and the Grenada Back-Arc Basin, East Caribbean Sea. J. Geophys. Res. Solid Earth 2021, 126, e2020JB020466. [CrossRef]

42. Bond, J.E.; Hamilton, C.A.; Godwin, R.L.; Ledford, J.M.; Starrett, J. Phylogeny, evolution, and biogeography of the North American trapdoor spider family Euctenizidae (Araneae: Mygalomorphae) and the discovery of a new 'endangered living fossil'along California's central coast. Insect Syst. Divers. 2020, 4, 2. [CrossRef]

43. Chousou-Polydouri, N.; Carmichael, A.; Szúts, T.; Saucedo, A.; Gillespie, R.; Griswold, C.; Wood, H.M. Giant Goblins above the waves at the southern end of the world: The biogeography of the spider family Orsolobidae (Araneae, Dysderoidea). J. Biogeogr. 2019, 46, 332-342. [CrossRef]

44. Rix, M.G.; Wilson, J.D.; Huey, J.A.; Hillyer, M.J.; Gruber, K.; Harvey, M.S. Diversification of the mygalomorph spider genus Aname (Araneae: Anamidae) across the Australian arid zone: Tracing the evolution and biogeography of a continent-wide radiation. Mol. Phylogenet. Evol. 2021, 160, 107127. [CrossRef]

45. Richardson, B.J. Evolutionary biogeography of Australian jumping spider genera (Araneae: Salticidae). Aust. J. Zool. 2020, 67, 162-172. [CrossRef]

46. Chamberland, L.; McHugh, A.; Kechejian, S.; Binford, G.J.; Bond, J.E.; Coddington, J.; Dolman, G.; Hamilton, C.A.; Harvey, M.S.; Kuntner, M.; et al. From Gondwana to GAARlandia: Evolutionary history and biogeography of ogre-faced spiders (Deinopis). J. Biogeogr. 2018, 45, 2442-2457. [CrossRef]

47. Hedges, S.B.; Hass, C.A.; Maxson, L.R. Caribbean biogeography: Molecular evidence for dispersal in West Indian terrestrial vertebrates. Proc. Natl. Acad. Sci. USA 1992, 89, 1909-1913. [CrossRef]

48. Betancur-R, R.; Hines, A.; Acero, P.A.; Ortí, G.; Wilbur, A.E.; Freshwater, D.W. Reconstructing the lionfish invasion: Insights into Greater Caribbean biogeography. J. Biogeogr. 2011, 38, 1281-1293. [CrossRef]

49. Klein, N.K.; Burns, K.J.; Hackett, S.J.; Griffiths, C.S. Molecular phylogenetic relationships among the wood warblers (Parulidae) and historical biogeography in the Caribbean basin. J. Caribb. Ornithol. 2004, 17, 3-17.

50. Ferrier, S.; Powell, G.V.N.; Richardson, K.S.; Manion, G.; Overton, J.M.; Allnutt, T.F.; Cameron, S.E.; Mantle, K.; Burgess, N.D.; Faith, D.P.; et al. Mapping more of terrestrial biodiversity for global conservation assessment. Bioscience 2004, 54, 1101-1109. [CrossRef] 
51. McHugh, A.; Yablonsky, C.; Binford, G.; Agnarsson, I. Molecular phylogenetics of Caribbean Micrathena (Araneae:Araneidae) suggests multiple colonisation events and single island endemism. Invertebr. Syst. 2014, 28, 337-349. [CrossRef]

52. Zhang, J.-X.; Maddison, W.P. Molecular phylogeny, divergence times and biogeography of spiders of the subfamily Euophryinae (Araneae: Salticidae). Mol. Phylogenet. Evol. 2013, 68, 81-92. [CrossRef] [PubMed]

53. Magalhães, I.L.F.; Santos, A.J. Phylogenetic analysis of Micrathena and Chaetacis spiders (Araneae: Araneidae) reveals multiple origins of extreme sexual size dimorphism and long abdominal spines. Zool. J. Linn. Soc. 2012, 166, 14-53. [CrossRef]

54. Levi, H.W. The spiny orb-weaver genera Micrathena and Chaetacis (Araneae: Araneidae). Los géneros de tejedoras de esferas espinosas Micrathena y Chaetacis (Araneae: Araneidae). Bull. Museum Comp. Zool. 1985, 150, 429-618.

55. Bukowski, T.C.; Christenson, T.E. Natural history and copulatory behavior of the spiny orbweaving spider Micrathena gracilis (Araneae, Araneidae). J. Arachnol. 1997, 307-320.

56. Bell, J.R.; Bohan, D.A.; Shaw, E.M.; Weyman, G.S. Ballooning dispersal using silk: World fauna, phylogenies, genetics and models. Bull. Entomol. Res. 2005, 95, 69-114. [CrossRef]

57. World Spider Catalog. World Spider Catalog. Version 18.5. National History Museum Bern. Available online: http://ws.nmbe.ch (accessed on 1 March 2018).

58. Scharff, N.; Coddington, J.A.; Blackledge, T.A.; Agnarsson, I.; Framenau, V.W.; Szúts, T.; Hayashi, C.Y.; Dimitrov, D. Phylogeny of the orb-weaving spider family Araneidae (Araneae: Araneoidea). Cladistics 2020, 36, 1-21. [CrossRef]

59. Agnarsson, I.; Maddison, W.P.; Avilés, L. The phylogeny of the social Anelosimus spiders (Araneae: Theridiidae) inferred from six molecular loci and morphology. Mol. Phylogenet. Evol. 2007, 43, 833-851. [CrossRef]

60. Kuntner, M.; Agnarsson, I. Biogeography and diversification of hermit spiders on Indian Ocean islands (Nephilidae: Nephilengys). Mol. Phylogenet. Evol. 2011, 59, 477-488. [CrossRef]

61. Simon, C.; Frati, F.; Beckenbach, A.; Crespi, B.; Liu, H.; Flook, P. Evolution, weighting, and phylogenetic utility of mitochondrial gene sequences and a compilation of conserved polymerase chain reaction primers. Ann. Entomol. Soc. Am. 1994, 87, 651-701. [CrossRef]

62. Hedin, M.C.; Maddison, W.P. A combined molecular approach to phylogeny of the jumping spider subfamily Dendryphantinae (Araneae: Salticidae). Mol. Phylogenet. Evol. 2001, 18, 386-403. [CrossRef] [PubMed]

63. Folmer, O.; Black, M.; Hoeh, W.; Lutz, R.; Vrijenhoek, R. DNA primers for amplification of mitochondrial cytochrome c oxidase subunit I from diverse metazoan invertebrates. Mol. Mar. Biol. Biotechnol. 1994, 3, 294-299. [PubMed]

64. White, T.J.; Bruns, T.; Lee, S.; Taylor, J. Amplification and direct sequencing of fungal ribosomal RNA genes for phylogenetics. PCR Protoc. Guid. Methods Appl. 1990, 18, 315-322.

65. Green, P. Phrap. Version 1.090518. Available online: http:// phrap.org (accessed on 19 June 2019).

66. Green, P.; Ewing, B. Phred. Version 0.020425c. Available online: http://phrap.org/othersoftware.html (accessed on 19 June 2019).

67. Maddison, D.R.; Maddison, W.P. Chromaseq: A Mesquite Package for Analyzing Sequence Chromatograms. Version 1.02011. Available online: http:/ / mesquiteproject.org/packages/chromaseq (accessed on 19 June 2019).

68. Maddison, W.P.; Maddison, D.R. Mesquite: A Modular System for Evolutionary Analysis. Version 3.6. Available online: http:/ / mesquiteproject.org (accessed on 19 June 2019).

69. Katoh, K.; Rozewicki, J.; Yamada, K.D. MAFFT online service: Multiple sequence alignment, interactive sequence choice and visualization. Brief. Bioinform. 2019, 20, 1160-1166. [CrossRef]

70. Lanfear, R.; Frandsen, P.B.; Wright, A.M.; Senfeld, T.; Calcott, B. PartitionFinder 2: New methods for selecting partitioned models of evolution for molecular and morphological phylogenetic analyses. Mol. Biol. Evol. 2017, 34, 772-773. [CrossRef]

71. Akaike, H. Information theory and an extension of the maximum likelihood principle. In Selected Papers of Hirotugu Akaike; Springer: Berlin/Heidelberg, Germany, 1998; pp. 199-213.

72. Ronquist, F.; Teslenko, M.; Van Der Mark, P.; Ayres, D.L.; Darling, A.; Höhna, S.; Larget, B.; Liu, L.; Suchard, M.A.; Huelsenbeck, J.P. MrBayes 3.2: Efficient Bayesian phylogenetic inference and model choice across a large model space. Syst. Biol. 2012, 61, 539-542. [CrossRef]

73. Miller, M.A.; Pfeiffer, W.; Schwartz, T. The CIPRES science gateway: A community resource for phylogenetic analyses. In Proceedings of the 2011 TeraGrid Conference: Extreme Digital Discovery, Salt Lake City, UT, USA, 18-21 July 2011; pp. 1-8.

74. Rambaut, A.; Drummond, A.J.; Xie, D.; Baele, G.; Suchard, M.A. Posterior summarization in Bayesian phylogenetics using Tracer 1.7. Syst. Biol. 2018, 67, 901. [CrossRef]

75. Bouckaert, R.; Vaughan, T.G.; Barido-Sottani, J.; Duchêne, S.; Fourment, M.; Gavryushkina, A.; Heled, J.; Jones, G.; Kühnert, D.; De Maio, N.; et al. BEAST 2.5: An advanced software platform for Bayesian evolutionary analysis. PLoS Comput. Biol. 2019, 15, e1006650. [CrossRef]

76. Kuntner, M.; Arnedo, M.A.; Trontelj, P.; Lokovšek, T.; Agnarsson, I. A molecular phylogeny of nephilid spiders: Evolutionary history of a model lineage. Mol. Phylogenet. Evol. 2013, 69, 961-979. [CrossRef] [PubMed]

77. Bidegaray-Batista, L.; Arnedo, M.A. Gone with the plate: The opening of the Western Mediterranean basin drove the diversification of ground-dweller spiders. BMC Evol. Biol. 2011, 11, 317. [CrossRef] [PubMed]

78. Matzke, N.J. BioGeoBEARS: BioGeography with Bayesian (and likelihood) evolutionary analysis in R Scripts. R Packag. 2013, 1, 2013.

79. Bartolini, C.; Buffler, R.T.; Blickwede, J.F. The Circum-Gulf of Mexico and the Caribbean: Hydrocarbon Habitats, Basin Formation, and Plate Tectonics, AAPG Memoir 79; American Association of Petroleum Geologists: Tulsa, OK, USA, 2003. 
80. Marshall, J.S. The geomorphology and physiographic provinces of Central America. Cent. Am. Geol. Resour. Hazards 2007, 1, 75-121.

81. Mann, P. Overview of the tectonic history of northern Central America. Geol. Soc. Am. Spec. Pap. 2007, 428, 1-19.

82. Randazzo, A.F.; Jones, D.S. The Geology of Florida; University Press of Florida: Gainesville, FL, USA, 1997.

83. Ree, R.H.; Sanmartín, I. Conceptual and statistical problems with the DEC+ J model of founder-event speciation and its comparison with DEC via model selection. J. Biogeogr. 2018, 45, 741-749. [CrossRef]

84. Ree, R.H.; Smith, S.A. Maximum Likelihood Inference of Geographic Range Evolution by Dispersal, Local Extinction, and Cladogenesis. Syst. Biol. 2008, 57, 4-14. [CrossRef]

85. Kozub, D.; Khmelik, V.; Shapoval, Y.; Chentsov, V.; Yatsenko, S.; Litovchenko, B.; Starykh, V. Helicon Focus Software. Available online: http:/ / heliconsoft.com (accessed on 26 January 2017).

86. Garrison, N.L.; Rodriguez, J.; Agnarsson, I.; Coddington, J.A.; Griswold, C.E.; Hamilton, C.A.; Hedin, M.; Kocot, K.M.; Ledford, J.M.; Bond, J.E. Spider phylogenomics: Untangling the Spider Tree of Life. PeerJ 2016, 4, e1719. [CrossRef]

87. Čandek, K.; Agnarsson, I.; Binford, G.J.; Kuntner, M. Global biogeography of Tetragnatha spiders reveals multiple colonization of the Caribbean. BioRxiv 2018. [CrossRef]

88. Peck, S.B. Aerial dispersal of insects between and to islands in the Galapagos Archipelago, Ecuador. Ann. Entomol. Soc. Am. 1994, 87, 218-224. [CrossRef]

89. Aldrich, J.R. Others Dispersal of the southern green stink bug, Nezara viridula (L.) (Heteroptera: Pentatomidae), by hurricane Hugo. Proc. Entomol. Soc. Washingt. 1990, 92, 757-759.

90. Zimmerman, E.C. Insects of Hawaii; University of Hawaii Press: Honolulu, HI, USA, 1948; Volume 7.

91. Oey, L.; Ezer, T.; Lee, H. Loop Current, rings and related circulation in the Gulf of Mexico: A review of numerical models and future challenges. Geophys. Monogr. Geophys. Union 2005, 161, 31.

92. Harrison, S.E.; Harvey, M.S.; Cooper, S.J.B.; Austin, A.D.; Rix, M.G. Across the Indian Ocean: A remarkable example of trans-oceanic dispersal in an austral mygalomorph spider. PLoS ONE 2017, 12, e0180139. [CrossRef]

93. Ceccarelli, F.S.; Opell, B.D.; Haddad, C.R.; Raven, R.J.; Soto, E.M.; Ramírez, M.J. Around the world in eight million years: Historical biogeography and evolution of the spray zone spider Amaurobioides (Araneae: Anyphaenidae). PLoS ONE 2016, 11, e0163740. [CrossRef] [PubMed]

94. Duque-Caro, H. Neogene stratigraphy, paleoceanography and paleobiogeography in northwest South America and the evolution of the Panama Seaway. Palaeogeogr. Palaeoclimatol. Palaeoecol. 1990, 77, 203-234. [CrossRef]

95. Coates, A.G.; Jackson, J.B.C.; Collins, L.S.; Cronin, T.M.; Dowsett, H.J.; Bybell, L.M.; Jung, P.; Obando, J.A. Closure of the Isthmus of Panama: The near-shore marine record of Costa Rica and western Panama. Geol. Soc. Am. Bull. 1992, 104, 814-828. [CrossRef]

96. Holcombe, T.L.; Moore, W.S. Paleocurrents in the eastern Caribbean: Geologic evidence and implications. Mar. Geol. 1977, 23, 35-56. [CrossRef]

97. Houle, A. Floating islands: A mode of long-distance dispersal for small and medium-sized terrestrial vertebrates. Divers. Distrib. 1998, 4, 201-216.

98. Lee, V.M.J.; Kuntner, M.; Li, D. Ballooning behavior in the golden orbweb spider Nephila pilipes (Araneae: Nephilidae). Front. Ecol. Evol. 2015, 3, 2. [CrossRef] 